\title{
Generalized ABC theorems for non-Archimedean entire functions of several variables in arbitrary characteristic
}

\author{
by \\ William Cherry (Denton, TX) and \\ Cristina Toropu (Albuquerque, NM)
}

1. Introduction. The well-known ABC theorem for polynomials, first proved by Stothers [31] and often called "Mason's theorem" [22], says that if $a+b=c$, where $a$ and $b$ are relatively prime univariate polynomials with at least one of the derivatives $a^{\prime}$ or $b^{\prime}$ not identically zero, then

$$
\max \{\operatorname{deg} a, \operatorname{deg} b, \operatorname{deg} c\} \leq \operatorname{deg} R(a b c)-1 .
$$

Here if $f$ is a polynomial, we use $R(f)$ to denote $f / \operatorname{gcd}\left(f, f^{\prime}\right)$. In characteristic zero, $\operatorname{deg} R(f)$ is simply the degree of the square free part of $f$, also called the degree of the radical of $f$, and is the number of distinct zeros of $f$ in an algebraically closed field containing the coefficients of $f$. We note that the above theorem immediately extends to polynomials of several variables by replacing the ordinary derivative with a partial derivative.

The existence of an appropriately analogous inequality for $c=a+b$ with $a, b$, and $c$ relatively prime integers is the famous $\mathrm{ABC}$ conjecture of Masser and Oesterlé (see [25]), which states that for each $\varepsilon>0$, there exists a constant $C(\varepsilon)$ such that

$$
\max \{|a|,|b|,|c|\} \leq C(\varepsilon) S(a b c)^{1+\varepsilon},
$$

where we use $S(a b c)$ to denote the square free part of $a b c$. The ABC conjecture for integers has spectacular consequences in number theory-see e.g. [16] and [21]. To date, in the case of integers, the best proven upper bounds

2000 Mathematics Subject Classification: 11D04, 11D88, 32P05.

Key words and phrases: ABC theorems, non-Archimedean entire functions, several variables, Hasse derivatives, vanishing sums, Mason's theorem, generalized Wronskians.

Partial financial support for this research was provided by a Faculty Research Grant from the University of North Texas and by the United States National Security Agency under Grant Number H98230-07-1-0037. The United States Government is authorized to reproduce and distribute preprints notwithstanding any copyright notation herein. 
on $\max \{|a|,|b|,|c|\}$ in terms of $S(a b c)$ are super-polynomial in $S(a b c)$-see e.g. [30].

The $\mathrm{ABC}$ theorem for polynomials has been generalized in a variety of directions, including: to sums in one-dimensional function fields by Mason [23], by Voloch [34] and by Brownawell and Masser [10], to sums of pairwise relatively prime polynomials of several variables by Shapiro and Sparer [29], to sums in higher-dimensional function fields by Hsia and Wang [18], and to quantum deformations of polynomials by Vaserstein [32]. Motivated by the analogy between Diophantine approximation and Nevanlinna theory [33], the $\mathrm{ABC}$ theorem has also been proven for complex entire functions by Van Frankenhuysen [13], [14] and for $p$-adic entire functions by $\mathrm{Hu}$ and Yang [19].

In a recent article, An and Manh [3] gave an ABC type theorem for $p$-adic entire functions of several variables $c=a+b$, but under some rather restrictive hypotheses, including the assumption that $a, b$, and $c$ have no common zeros, which in several variables is a much stronger assumption than simply supposing that $a, b$, and $c$ are pairwise relatively prime in the ring of entire functions. The purpose of this article is to prove general $\mathrm{ABC}$ theorems for sums

$$
f_{n}=f_{0}+\cdots+f_{n-1}
$$

of non-Archimedean entire functions on affine $m$-space $\mathbb{A}^{m}$ in arbitrary characteristic analogous to the existing theorems for several-variable polynomials. We do this for two reasons. First, we illustrate that if the existing polynomial proofs for $\mathrm{ABC}$ theorems are correctly interpreted, then they provide immediate proofs for the analogous statements for non-Archimedean entire functions without the need for any fundamentally new ideas or for additional technical assumptions as in [3], with the exception of one slight subtlety discussed just before Corollary 6.5 below. Second, Cherry and Ye [12] developed a several-variable non-Archimedean Nevanlinna theory in a form that was intended to be easy to use. However, in applications, it is often convenient to work with, or at least to think in terms of, truncated counting functions, which were not discussed in [12]. Thus, our second purpose is to illustrate how to define and work with truncated counting functions in several variables and in positive characteristic; this is well-known to the experts, but we thought it helpful to illustrate here for the novice's benefit.

The plan of this paper is as follows. In Section 2 we set up some notation and recall some basic facts we will need. We also prove the most basic form of the ABC theorem in Section 2, so readers only interested in the basic idea do not need to read past Section 2. In Section 3, we recall the notion of Hasse derivative and generalized Wronskians necessary to work in positive characteristic and in several variables. In Section 4, we discuss how 
to define truncated counting functions in positive characteristic. We recall some linear algebra from Brownawell and Masser [10] in Section 5. Finally, in Section 6, we derive our general $\mathrm{ABC}$ theorems and indicate how various $\mathrm{ABC}$ theorems in the literature can be derived as corollaries. Our method of proof is essentially that of $\mathrm{Hu}$ and Yang [20], who adapted the argument of Brownawell and Masser [10] to the context of non-Archimedean entire functions of one variable. Our presentation in Section 6 was also influenced by the recent work of De Bondt [5], who formulated generalized ABC theorems for complex polynomials in several variables from which the other various versions in the literature can be derived.

2. Preliminaries, notation and warm-up. We will find it convenient to use some Nevanlinna notation. We will use [12] as our basic reference and mostly follow the notation there, although here we will not assume characteristic zero, as was done in [12].

Throughout, $\mathbb{F}$ will denote an algebraically closed field complete with respect to a non-Archimedean absolute value | |. We make no assumption about the characteristic of $\mathbb{F}$. Let $\mathbb{F}^{\times}$denote $\mathbb{F} \backslash\{0\}$, and let $\left|\mathbb{F}^{\times}\right|$be the subset of the positive real numbers defined by

$$
\left|\mathbb{F}^{\times}\right|=\left\{|a|: a \in \mathbb{F}^{\times}\right\} .
$$

Let $\mathbb{F}^{m}$ denote the $m$ th Cartesian product of $\mathbb{F}$, which is the set of $\mathbb{F}$ points of affine $m$-space $\mathbb{A}^{m}$. By an entire function on $\mathbb{A}^{m}$ or $\mathbb{F}^{m}$, we mean a formal power series in $m$ variables with coefficients in $\mathbb{F}$ and with infinite radius of convergence. We will use $\mathcal{E}_{m}$ to denote the ring of entire functions on $\mathbb{A}^{m}$.

If $z_{1}, \ldots, z_{m}$ are $\mathbb{F}$-valued variables, we use $z$ to refer collectively to the $m$-tuple $\left(z_{1}, \ldots, z_{m}\right)$. When convenient, we will use multi-index notation. If $\gamma=\left(\gamma_{1}, \ldots, \gamma_{m}\right)$ is a multi-index, i.e., an $m$-tuple of non-negative integers, then by definition

$$
z^{\gamma}=z_{1}^{\gamma_{1}} \cdots z_{m}^{\gamma_{m}}, \quad|\gamma|=\gamma_{1}+\cdots+\gamma_{m}, \quad \partial^{\gamma} f=\frac{\partial^{|\gamma|} f}{\partial z^{\gamma}} .
$$

Similarly, if $\mathbf{r}=\left(r_{1}, \ldots, r_{m}\right)$ is an $m$-tuple of non-negative real numbers, we define

$$
\mathbf{r}^{\gamma}=r_{1}^{\gamma_{1}} \cdots r_{m}^{\gamma_{m}}
$$

We can therefore write an entire function $f$ in $\mathcal{E}_{m}$ as

$$
f(z)=\sum_{\gamma} a_{\gamma} z^{\gamma}
$$

where $a_{\gamma}$ are in $\mathbb{F}$, and for all $m$-tuples of non-negative real numbers $\mathbf{r}$,

$$
\lim _{|\gamma| \rightarrow \infty}\left|a_{\gamma}\right| \mathbf{r}^{\gamma}=0 \text {. }
$$


We recall that to each $m$-tuple $\mathbf{r}=\left(r_{1}, \ldots, r_{m}\right)$ of non-negative real numbers, we can associate a non-Archimedean absolute value ||$_{\mathbf{r}}$ on the ring $\mathcal{E}_{m}$ by defining

$$
|f|_{\mathbf{r}}=\sup _{\gamma}\left|a_{\gamma}\right| \mathbf{r}^{\gamma}
$$

where as above $f$ in $\mathcal{E}_{m}$ is given by the power series expansion

$$
f(z)=\sum_{\gamma} a_{\gamma} z^{\gamma} .
$$

The non-trivial thing that needs to be checked is that if $f$ and $g$ are two elements of $\mathcal{E}_{m}$, then $|f g|_{\mathbf{r}}=|f|_{\mathbf{r}}|g|_{\mathbf{r}}$. When all the $r_{j}$ equal 1 this is worked out, for instance, in $[6, \S 5.1 .2]$. In general, by extending the field $\mathbb{F}$ if necessary, we may assume the $r_{j}$ are elements of $\left|\mathbb{F}^{\times}\right|$and then reduce to the case when all the $r_{j}$ are 1 by an affine rescaling of the variables.

For our purposes, we only need to consider those $\mathbf{r}$ for which all the $r_{j}$ are equal, or in other words $m$-tuples of the form $\mathbf{r}=(r, \ldots, r)$. We will denote the associated absolute value on $\mathcal{E}_{m}$ by ||$_{r}$. Clearly, we have:

Proposition 2.1. If $f$ is in $\mathcal{E}_{m}$, then $|f|_{r}$ is a non-decreasing function of $r$.

If $f$ happens to be a polynomial of degree $d$, then we easily see that as $r \rightarrow \infty$,

$$
\log |f|_{r}=d \log r+O(1) .
$$

Thus, in our ABC theorems for entire functions, $\log |f|_{r}$ will play the role played by the degree in the case of polynomials on the left-hand side of the inequalities.

We will make use of the following observation on several occasions:

COROLlary 2.2. If $f, g$ and $h$ are elements of $\mathcal{E}_{m}$ such that $f=g h$ and if $r_{0}>0$, then for all $r \geq r_{0}$,

$$
\log |g|_{r} \leq \log |f|_{r}+O(1) .
$$

Proof. By the multiplicativity of ||$_{r}$ and Proposition 2.1,

$$
\log |f|_{r}=\log |g|_{r}+\log |h|_{r} \geq \log |g|_{r}+\log |h|_{r_{0}},
$$

which gives the required inequality.

We recall the elementary

Lemma 2.3 (Logarithmic derivative lemma). Let $f$ be an entire function in $\mathcal{E}_{m}$ and let $\gamma$ be a multi-index. Then

$$
\left|\partial^{\gamma} f\right|_{r} \leq \frac{|f|_{r}}{r|\gamma|}
$$


Proof. Differentiate the power series defining $f$ and use the fact that $|k| \leq 1$ for any integer $k$.

We need to make use of some ring-theoretic properties of $\mathcal{E}_{m}$. The reader can see [11] for a detailed treatment.

As usual, we will call an element $P$ of $\mathcal{E}_{m}$ irreducible if whenever we write $P=f g$ with $f$ and $g$ in $\mathcal{E}_{m}$, then at least one of $f$ or $g$ is a unit in $\mathcal{E}_{m}$. As is well-known, the only units in $\mathcal{E}_{m}$ are the non-zero constant functions-see e.g. [12, Cor. 2.4].

Proposition 2.4. Let $P$ be an irreducible element of $\mathcal{E}_{m}$ and let $j$ be an integer between 1 and $m$. If $P$ divides $\partial P / \partial z_{j}$, then $\partial P / \partial z_{j} \equiv 0$.

Proof. Suppose

$$
\frac{\partial P}{\partial z_{j}}=P g
$$

for some $g$ in $\mathcal{E}_{m}$. Then

$$
\left|\frac{\partial P}{\partial z_{j}}\right|_{r}=|P|_{r}|g|_{r}
$$

From Lemma 2.3, $|g|_{r} \leq 1 / r$, and so by Proposition $2.1,|g|_{r} \equiv 0$.

Suppose $f$ and $g$ are non-constant elements of $\mathcal{E}_{m}$ such that $g$ divides $f$ in $\mathcal{E}_{m}$. Then $f$ and $g$ can also be considered as analytic functions on the closed ball of radius $r$, i.e.,

$$
\mathbf{B}^{m}(r)=\left\{\left(z_{1}, \ldots, z_{m}\right) \in \mathbb{F}^{m}: \max \left|z_{j}\right| \leq r\right\} .
$$

For large $r$, the function $g$ will have zeros inside the ball (again see [12, Cor. 2.4]), and hence will not be a unit in the ring of analytic functions on the ball $\mathbf{B}^{m}(r)$. These rings are Tate algebras when $r \in\left|\mathbb{F}^{\times}\right|$, and hence factorial $\left[6, \S 5.2 .6\right.$, Th. 1]. Thus, $\mathcal{E}_{m}$ is a subring of a factorial ring in which $g$ is not a unit, and hence some power of $g$ will not divide $f$ in $\mathcal{E}_{m}$. Therefore, one can speak of the multiplicity with which an entire function $g$ divides another entire function $f$. Although $\mathcal{E}_{m}$ itself is not factorial, the notion of "greatest common divisor" does make sense in $\mathcal{E}_{m}$; see [11] or the appendix of [12]. Of course, greatest common divisors are only defined up to units, hence multiplicative constants. Given two entire functions $f_{1}$ and $f_{2}$, when we write something like $g=\operatorname{gcd}\left(f_{1}, f_{2}\right)$, we mean picking any function $g$ which is a greatest common divisor of $f_{1}$ and $f_{2}$. Hence $g$ is only well-defined up to a choice of multiplicative constant.

Since we have greatest common divisors, we can define a good notion of the "radical" or the "square free part" of an entire function, at least in characteristic zero. The definition we give here will not be the square free part in positive characteristic, but will be the suitable thing to put on the right-hand side in our basic $\mathrm{ABC}$ theorem. We will discuss the existence of 
the square-free part of an analytic function in positive characteristic in a later section.

Proposition 2.5. Let $f$ be an entire function in $\mathcal{E}_{m}$. For each $j$ from 1 to $m$, define

$$
g_{j}=\operatorname{gcd}\left(f, \frac{\partial f}{\partial z_{j}}\right) \quad \text { and } \quad h_{j}=\frac{f}{g_{j}} .
$$

Let $R(f)$ be the least common multiple of the $h_{j}$. Then:

(i) $R(f)$ divides $f$;

(ii) for any non-constant $g$ in $\mathcal{E}_{m}, g^{2}$ does not divide $R(f)$;

(iii) if $P$ is an irreducible element of $\mathcal{E}_{m}$ that divides $f$, then $P$ also divides $R(f)$ if and only if the multiplicity to which $P$ divides $f$ is not divisible by the characteristic of $\mathbb{F}$.

We will call $R(f)$ as defined in Proposition 2.5 the radical of $f$.

Proof. For (i), note that because each of the $h_{j}$ divides $f$, it is clear that their least common multiple $R(f)$ also divides $f$.

To show (ii), suppose $g$ is a non-constant element of $\mathcal{E}_{m}$ such that $g^{2}$ divides $R(f)$. Then $g^{2}$ must also divide $f$ since $R(f)$ divides $f$. Let $s \geq 2$ be the largest integer such that $g^{s}$ divides $f$. Then $f=g^{s} \tilde{f}$, where $\tilde{f}$ is an element of $\mathcal{E}_{m}$ not divisible by $g$. Because

$$
\frac{\partial f}{\partial z_{j}}=\tilde{f} s g^{s-1} \frac{\partial g}{\partial z_{j}}+g^{s} \frac{\partial \widetilde{f}}{\partial z_{j}}
$$

we see that $g^{s-1}$ divides $g_{j}$ for all $j$, and hence $g^{2}$ does not divide $h_{j}$ for any $j$. Thus, $g^{2}$ cannot divide $R(f)$.

To show (iii), let $P$ be a non-constant irreducible element of $\mathcal{E}_{m}$ that divides $f$. Let $s$ be the largest integer such that $P^{s}$ divides $f$. Then $f=P^{s} \widetilde{f}$ with $\widetilde{f}$ relatively prime to $P$. If $\partial P / \partial z_{j} \equiv 0$ for all $j$, then because we have assumed that $P$ is non-constant, it follows that $P$ is a $p$ th power and $\mathbb{F}$ has positive characteristic $p$. But then $P$ would not be irreducible, and so there must exist some $j$ such that $\partial P / \partial z_{j} \not \equiv 0$. Because

$$
\frac{\partial f}{\partial z_{j}}=s P^{s-1} \frac{\partial P}{\partial z_{j}} \widetilde{f}+P^{s} \frac{\partial \widetilde{f}}{\partial z_{j}},
$$

we conclude from Proposition 2.4 that $P^{s}$ divides $\partial f / \partial z_{j}$ if and only if $s=0$ in $\mathbb{F}$.

We will now state and prove the most basic version of an $\mathrm{ABC}$ theorem for non-Archimedean entire functions of several variables. We feel that discussing this basic case here will help the reader see the main ideas behind what we will do. 
Theorem 2.6 (Basic ABC theorem). Let $f_{2}=f_{0}+f_{1}$ be entire functions such that $f_{0}$ and $f_{1}$ are relatively prime in $\mathcal{E}_{m}$. If $\mathbb{F}$ has characteristic zero, assume that at least one of $f_{0}$ or $f_{1}$ is non-constant. If $\mathbb{F}$ has positive characteristic $p$, then assume that at least one of $f_{0}$ or $f_{1}$ is not a pth power in $\mathcal{E}_{m}$. Let $r_{0}>0$. Then, for $r \geq r_{0}$,

$$
\max _{0 \leq i \leq 2} \log \left|f_{i}\right|_{r} \leq \log \left|R\left(f_{0} f_{1} f_{2}\right)\right|_{r}-\log r+O(1) .
$$

Proof. We follow the standard polynomial proof, as given for instance in [32], mutatis mutandis. Without loss of generality assume that $f_{0}$ is nonconstant and if $\mathbb{F}$ has positive characteristic $p$ that $f_{0}$ is not a $p$ th power in $\mathcal{E}_{m}$. This implies there exists a non-constant irreducible element $P_{0}$ in $\mathcal{E}_{m}$ that divides $f_{0}$ to a multiplicity $s_{0}$ not divisible by the characteristic of $\mathbb{F}$. Without loss of generality, assume that $\partial P_{0} / \partial z_{1} \not \equiv 0$. Consider the Wronskian determinant,

$$
W=\operatorname{det}\left(\begin{array}{cc}
f_{0} & f_{1} \\
\frac{\partial f_{0}}{\partial z_{1}} & \frac{\partial f_{1}}{\partial z_{1}}
\end{array}\right)=\operatorname{det}\left(\begin{array}{cc}
f_{0} & f_{2} \\
\frac{\partial f_{0}}{\partial z_{1}} & \frac{\partial f_{2}}{\partial z_{1}}
\end{array}\right)=\operatorname{det}\left(\begin{array}{cc}
f_{2} & f_{1} \\
\frac{\partial f_{2}}{\partial z_{1}} & \frac{\partial f_{1}}{\partial z_{1}}
\end{array}\right),
$$

where the first equality defines $W$ in $\mathcal{E}_{m}$ and the second two equalities follow from $f_{2}=f_{0}+f_{1}$.

We first claim that $W \not \equiv 0$. Indeed, if $W \equiv 0$, then

$$
f_{0} \frac{\partial f_{1}}{\partial z_{1}}=f_{1} \frac{\partial f_{0}}{\partial z_{1}} \text {. }
$$

Because $P_{0}^{s_{0}}$ divides $f_{0}$ and does not divide $\partial f_{0} / \partial z_{1}$, this would imply that $P_{0}$ divides $f_{1}$. But $f_{0}$ and $f_{1}$ were assumed relatively prime, and hence $W \not \equiv 0$.

Let $F=f_{0} f_{1} f_{2}, G=\operatorname{gcd}\left(F, \partial F / \partial z_{1}\right)$, and $H=F / G$. Then by definition $H$ divides $R\left(f_{0} f_{1} f_{2}\right)$, and so

$$
\log |H|_{r} \leq \log \left|R\left(f_{0} f_{1} f_{2}\right)\right|_{r}+O(1)
$$

for $r \geq r_{0}$ by Corollary 2.2. We also claim that $G$ divides $W$. Indeed, suppose that $P$ is an irreducible element that divides $G$. Then $P$ divides $F$ and so it divides one of the $f_{i}$ and hence exactly one of the $f_{i}$ since the $f_{i}$ are relatively prime. Thus, suppose that $P$ divides $f_{i}$ and hence $F$ with multiplicity $s$. Then $P^{s-1}$ divides $\partial f_{i} / \partial z_{1}$ and hence also $W$. If $P^{s}$ also divides $G$ and hence $\partial F / \partial z_{1}$, then either $s$ is divisible by the characteristic of $\mathbb{F}$ or $\partial P / \partial z_{1}=0$. But in either of these cases, $P^{s}$ also divides $\partial f_{i} / \partial z_{1}$, and so $P^{s}$ also divides $W$. Thus, $G$ divides $W$ as claimed. Again applying Corollary 2.2, we see that for $r \geq r_{0}$,

$$
\log |G|_{r} \leq \log |W|_{r}+O(1) .
$$


By Lemma 2.3,

$$
\left|f_{i} \frac{\partial f_{j}}{\partial z_{1}}\right|_{r} \leq \frac{\left|f_{i} f_{j}\right|_{r}}{r}
$$

and hence using each of the three determinants defining $W$,

$$
\log |W|_{r} \leq \log \min \left\{\left|f_{0} f_{1}\right|_{r},\left|f_{0} f_{2}\right|_{r},\left|f_{1} f_{2}\right|_{r}\right\}-\log r .
$$

Hence,

$$
\begin{aligned}
\log \max \left|f_{i}\right|_{r} & =\log \left|f_{0}\right|_{r}+\log \left|f_{1}\right|_{r}+\log \left|f_{2}\right|_{r}-\log \min _{0 \leq i<j \leq 2}\left|f_{i} f_{j}\right|_{r} \\
& =\log |F|_{r}-\log \min _{0 \leq i<j \leq 2}\left|f_{i} f_{j}\right|_{r} \\
& =\log |H|_{r}+\log |G|_{r}-\log \min _{0 \leq i<j \leq 2}\left|f_{i} f_{j}\right|_{r} \\
& \leq \log |R(F)|_{r}+\log |W|_{r}-\log \min _{0 \leq i<j \leq 2}\left|f_{i} f_{j}\right|_{r}+O(1) \\
& \leq \log |R(F)|_{r}-\log r+O(1)
\end{aligned}
$$

for $r \geq r_{0}$.

We conclude this section with a discussion of counting functions. For a polynomial in one variable, it is a simple matter to count the zeros, with or without multiplicity, because they are finite in number. For a one-variable convergent power series, the zeros are discrete, so one can create a counting function by counting them up to a certain size and then seeing how the number of zeros grows as the maximum size considered is allowed to grow. This is in complete analogy to Nevanlinna's notion of a counting function to count the number of zeros of a complex entire or meromorphic function.

For several-variable polynomials, one generally does not try to "count" zeros. Rather, one counts irreducible factors, usually weighted by the degree of the irreducible factor. For complex holomorphic functions of several variables, including the case of complex polynomials, one can define counting functions in a very geometric way by integrating certain differential forms over the irreducible components of the zero divisor of the function; see e.g. [24] or [28].

One approach to defining non-Archimedean counting functions in several variables is the approach initiated by Hà Huy Khoái [17] and used by An and Manh [1]-[3]. Although this approach is, in principle, aesthetically pleasing because of its definition in terms of the geometry of the Newton polytope associated to a several-variable power series, in practice, working with counting functions defined in this way seems to be rather difficult and not to produce particularly aesthetic proofs. For instance, the difficulty in working with this notion of counting function seems to have something to do with An and Manh's need for some of their restrictive hypotheses in [3]. Moreover, working with this definition seems to obscure connections 
to proofs of similar results for polynomials. In [12], Cherry and Ye preferred not to give an a priori natural definition of counting function, but rather first proved [12, Lem. 2.3] that starting with a power series of several variables, the counting functions of the one-variable power series obtained by restricting to a sufficiently generic line through the origin do not depend on the generic line chosen and can be expressed in terms of the power series coefficients. The pay-back for doing this work first before giving what may seem like an unnatural definition for the counting function is that one can then in a relatively straightforward manner connect Cherry and Ye's notion of counting function with $|f|_{r}$ through a Poisson-Jensen-Green type formula [12, Th. 3.1]. Then one can work with $|f|_{r}$ in a relatively straightforward manner and in close analogy with how one would naturally work with a several-variable polynomial.

Suppose

$$
f=\sum_{\gamma} a_{\gamma} z^{\gamma}
$$

is an entire function on $\mathbb{A}^{m}$. As earlier in this section, let $r>0$ and let $\mathbf{r}=(r, \ldots, r)$. Cherry and Ye define the unintegrated counting function of zeros of $f$ by

$$
n_{f}(0, r)=\sup \left\{|\gamma|:\left|a_{\gamma}\right| \mathbf{r}^{\gamma}=|f|_{r}\right\} .
$$

This is the number of zeros, counting multiplicity, that $f$ has on a sufficiently generic line through the origin with $\max \left|z_{j}\right| \leq r$. Also, define

$$
n_{f}(0,0)=\lim _{r \rightarrow 0} n_{f}(0, r)=\min \left\{|\gamma|: a_{\gamma} \neq 0\right\} .
$$

As is typical in Nevanlinna theory, it is more convenient to work with the integrated counting function of zeros

$$
N_{f}(0, r)=n_{f}(0,0) \log r+\int_{0}^{r}\left(n_{f}(0, t)-n_{f}(0,0)\right) \frac{d t}{t} .
$$

Immediately from the definition we see that if $f$ is a non-constant entire function, then for $r \geq 1$,

$$
\log r \leq N_{f}(0, r)+O(1) .
$$

Cherry and Ye's Poisson-Jensen-Green formula [12, Th. 3.1] then says that there exists a constant $C_{f}$ depending on $f$ but not on $r$ such that

$$
N_{f}(0, r)=\log |f|_{r}+C_{f}
$$

for all $r$. These counting functions count zeros of $f$ with multiplicity.

The following proposition for counting functions corresponds to Corollary 2.2 . 
Proposition 2.7. Let $f=g h$ be entire functions. Then:

(i) $n_{f}(0, r)=n_{g}(0, r)+n_{h}(0, r)$ for all $r \geq 0$;

(ii) $N_{f}(0, r)=N_{g}(0, r)+N_{h}(0, r)$ for all $r \geq 0$;

(iii) $N_{f}(0, r) \geq N_{g}(0, r)$ for all $r \geq 1$.

Proof. The equality in (ii) follows immediately from (i) and the definition of the integrated counting functions. The inequality in (iii) follows from (ii) and the fact that $N_{h}(0, r) \geq 0$ if $r \geq 1$. Thus, we need to show (i). To do so, let

$$
f(z)=\sum_{\alpha} a_{\alpha} z^{\alpha}, \quad g(z)=\sum_{\beta} b_{\beta} z^{\beta}, \quad h(z)=\sum_{\gamma} c_{\gamma} z^{\gamma} .
$$

We leave the case $r=0$ for the reader. Let $r>0$. Let $\beta_{0}$ and $\gamma_{0}$ be the largest multi-indices in the graded lexicographical ordering such that

$$
\left.\left|b_{\beta_{0}}\right|\right|^{\left|\beta_{0}\right|}=|g|_{r} \quad \text { and } \quad\left|c_{\gamma_{0}}\right| r^{\left|\gamma_{0}\right|}=|h|_{r}
$$

respectively. By definition, $n_{g}(0, r)=\left|\beta_{0}\right|$ and $n_{h}(0, r)=\left|\gamma_{0}\right|$. Therefore for $|\alpha|>\left|\beta_{0}\right|+\left|\gamma_{0}\right|$, we have

$$
\left|a_{\alpha}\right| r^{|\alpha|} \leq \max _{\beta+\gamma=\alpha}\left|b_{\beta}\right| r^{|\beta|}\left|c_{\gamma}\right| r^{|\gamma|}<|g|_{r}|h|_{r}=|f|_{r},
$$

where the second inequality follows from the fact that if

$$
|\beta|+|\gamma|=|\alpha|>\left|\beta_{0}\right|+\left|\gamma_{0}\right|,
$$

then we must have

$$
|\beta|>\left|\beta_{0}\right| \text { or }|\gamma|>\left|\gamma_{0}\right| \text {. }
$$

On the other hand, if we consider $\alpha_{0}=\beta_{0}+\gamma_{0}$, then

$$
\alpha_{0}=\sum_{\beta+\gamma=\alpha_{0}} b_{\beta} c_{\gamma} \text {. }
$$

If $\beta \neq \beta_{0}$ (and so $\gamma \neq \gamma_{0}$ ), then either $\beta$ comes after $\beta_{0}$ or $\gamma$ comes after $\gamma_{0}$ in the graded lexicographical ordering, which means

$$
\left|b_{\beta}\right| r^{|\beta|}\left|c_{\gamma}\right| r^{|\gamma|}<\left|b_{\beta_{0}}\right| r^{\left|\beta_{0}\right|}\left|c_{\gamma_{0}}\right| r^{\left|\gamma_{0}\right|}
$$

and so

$$
\left|a_{\alpha_{0}}\right| r^{\left|\alpha_{0}\right|}=\left|b_{\beta_{0}}\right| r^{\left|\beta_{0}\right|}\left|c_{\gamma_{0}}\right| r^{\left|\gamma_{0}\right|}=|g|_{r}|h|_{r}=|f|_{r} .
$$

Thus,

$$
n_{f}(0, r)=\left|\alpha_{0}\right|=\left|\beta_{0}\right|+\left|\gamma_{0}\right|=n_{g}(0, r)+n_{h}(0, r) .
$$

In [12], Cherry and Ye did not discuss truncated counting functions, where zeros are counted without multiplicity or with their multiplicities "truncated" to a certain level. In complex Nevanlinna theory, since one has a natural geometric definition for counting functions defined as integrals over irreducible components of an analytic divisor, it is straightforward to define truncated counting functions. Since Cherry and Ye's definition of counting 
functions is given in terms of power series coefficients, it is clear that there will be no obvious definition of truncated counting functions in terms of the power series coefficients. Instead, we use the nice ring-theoretic properties of $\mathcal{E}_{m}$ discussed above, and in characteristic zero simply define truncated counting functions by

$$
n_{f}^{(1)}(0, r)=n_{R(f)}(0, r) \quad \text { and } \quad N_{f}^{(1)}(0, r)=N_{R(f)}(0, r),
$$

where, as before, $R(f)$ denotes the radical of $f$. Note that although $R(f)$ is only defined up to a multiplicative constant, $n^{(1)}$ and $N^{(1)}$ are well-defined. In characteristic zero, Proposition 2.5 justifies calling the counting functions of the radical the "truncated" counting function for $f$ because each irreducible factor of $f$ appears with multiplicity one in $R(f)$. In positive characteristic, $N_{R(f)}$ might be called "overly truncated" because it completely ignores all irreducible factors of $f$ which appear with multiplicity divisible by the characteristic. We will see in Section 4 how to define truncated counting functions in positive characteristic that include all irreducible factors. However, as we saw in Theorem 2.6, in positive characteristic we can sometimes give lower bounds on these overly truncated counting functions.

We complete this section by pointing out that Boutabaa and Escassut $[7,8]$ were the first to work out one-variable non-Archimedean Nevanlinna theory in positive characteristic. Their work also highlights that in working with Nevanlinna theory in positive characteristic, one may often ignore zeros whose multiplicity is divisible by the characteristic.

3. Hasse derivatives and generalized Wronskians. If $\mathbb{F}$ has characteristic zero, then a formal power series $f$ in the variables $z=\left(z_{1}, \ldots, z_{m}\right)$ is non-constant if and only if at least one of its formal partial derivatives $\partial f / \partial z_{j}$ is not identically zero. By contrast, if $\mathbb{F}$ has positive characteristic $p$, then any formal power series in $z^{p}=\left(z_{1}^{p}, \ldots, z_{m}^{p}\right)$ is such that all its partial derivatives $\partial^{\gamma} f$ are identically zero for all $|\gamma|>0$. Also, if $\mathbb{F}$ has positive characteristic $p$, then if $\gamma$ is a multi-index such that $\gamma_{i} \geq p$ for some $i$, then $\partial^{\gamma} f=0$ for all $f$. Therefore, we will introduce a modification of the standard derivative, known as the Hasse derivative, which is more useful in positive characteristic.

If $\alpha=\left(\alpha_{1}, \ldots, \alpha_{m}\right)$ and $\beta=\left(\beta_{1}, \ldots, \beta_{m}\right)$ are multi-indices, we use $\alpha+\beta$ to denote the multi-index

$$
\alpha+\beta=\left(\alpha_{1}+\beta_{1}, \ldots, \alpha_{m}+\beta_{m}\right) .
$$

We say that $\alpha \geq \beta$ if $\alpha_{i} \geq \beta_{i}$ for all $i$ from 1 to $m$. Note that this notion of $\geq$ is not a total ordering on the set of multi-indices and is not the graded lexicographical ordering that was used in the proof of Proposition 2.7. If 
$\alpha \geq \beta$, we use $\alpha-\beta$ to denote the multi-index

$$
\alpha-\beta=\left(\alpha_{1}-\beta_{1}, \ldots, \alpha_{m}-\beta_{m}\right) .
$$

Also, if $\alpha \geq \beta$, define the multinomial coefficient $\left(\begin{array}{l}\alpha \\ \beta\end{array}\right)$ by

$$
\left(\begin{array}{l}
\alpha \\
\beta
\end{array}\right)=\left(\begin{array}{c}
\alpha_{1} \\
\beta_{1}
\end{array}\right) \cdots\left(\begin{array}{c}
\alpha_{m} \\
\beta_{m}
\end{array}\right)
$$

where the $\left(\begin{array}{c}\alpha_{i} \\ \beta_{i}\end{array}\right)$ are the standard binomial coefficients. Given a formal power series

$$
f=\sum_{\alpha} a_{\alpha} z^{\alpha}
$$

and a multi-index $\gamma$, we define the Hasse derivative of multi-index $\gamma$ of $f$, which we will denote $D^{\gamma} f$, to be the formal power series defined by

$$
D^{\gamma} f=\sum_{\alpha \geq \gamma}\left(\begin{array}{l}
\alpha \\
\gamma
\end{array}\right) a_{\alpha} z^{\alpha-\gamma} .
$$

Note that if $\gamma=(0, \ldots, 0)$, then $D^{\gamma} f=f$, and that if $|\gamma|=1$, then $D^{\gamma} f=\partial^{\gamma} f$. Given a $j$ from 1 to $m$ and a positive integer $k$, we will use $D_{j}^{k}$ as a short-hand notation for $D^{\gamma} f$ where $\gamma=\left(\gamma_{0}, \ldots, \gamma_{m}\right)$ with $\gamma_{j}=k$ and $\gamma_{i}=0$ for $i \neq j$.

Because the multinomial coefficients $\left(\begin{array}{l}\alpha \\ \gamma\end{array}\right)$ are integers and hence have non-Archimedean absolute value at most 1 , we see that if $\mathbf{r}=\left(r_{1}, \ldots, r_{m}\right)$ is an $m$-tuple of non-negative real numbers such that

$$
\lim _{|\alpha| \rightarrow \infty}\left|a_{\alpha}\right| \mathbf{r}^{\alpha}=0,
$$

then

$$
\lim _{|\alpha| \rightarrow \infty}\left|\left(\begin{array}{c}
\alpha \\
\gamma
\end{array}\right) a_{\alpha}\right| \mathbf{r}^{\alpha-\gamma} \leq \frac{1}{\mathbf{r}^{\gamma}} \lim _{|\alpha| \rightarrow \infty}\left|a_{\alpha}\right| \mathbf{r}^{\alpha}=0,
$$

and so we see that if $f$ is in $\mathcal{E}_{m}$, then $D^{\gamma} f$ is also in $\mathcal{E}_{m}$.

Clearly,

$$
\partial^{\gamma} f=\gamma ! D^{\gamma} f, \quad \text { where } \gamma !=\gamma_{1} ! \cdots \gamma_{m} ! .
$$

Thus, in characteristic zero, the Hasse derivatives are just constant multiples of the ordinary derivatives, and so one sees immediately that they have similar properties to those of the ordinary partial derivative. In positive characteristic, one must check these.

Proposition 3.1. The Hasse derivatives have the following basic properties:

(i) $D^{\alpha}[f+g]=D^{\alpha} f+D^{\alpha} g$;

(ii) $D^{\alpha}[f g]=\sum_{\beta+\gamma=\alpha} D^{\beta} f D^{\gamma} g$;

(iii) $D^{\alpha} D^{\beta} f=\left(\begin{array}{c}\alpha+\beta \\ \beta\end{array}\right) D^{\alpha+\beta} f$; 
(iv) if $\mathbb{F}$ has positive characteristic $p$ and $s \geq 0$ is an integer, then

$$
D_{i}^{p^{s}} f^{p^{s}}=\left(D_{i} f\right)^{p^{s}} \text {. }
$$

Proof. Property (i) is obvious. To check property (ii), write out both sides and compare like powers of $z$. What is needed for equality is that for multi-indices $\delta$ and $\varepsilon$ with $\delta \geq \beta$ and $\varepsilon \geq \gamma$, one has

$$
\sum_{\beta+\gamma=\alpha}\left(\begin{array}{l}
\delta \\
\beta
\end{array}\right)\left(\begin{array}{l}
\varepsilon \\
\gamma
\end{array}\right)=\left(\begin{array}{c}
\delta+\varepsilon \\
\alpha
\end{array}\right)
$$

which is nothing other than Vandermonde's identity. To check property (iii), one uses the elementary observation that for $\gamma \geq \alpha+\beta$,

$$
\left(\begin{array}{c}
\alpha+\beta \\
\beta
\end{array}\right)\left(\begin{array}{c}
\gamma \\
\alpha+\beta
\end{array}\right)=\left(\begin{array}{c}
\gamma \\
\alpha
\end{array}\right)\left(\begin{array}{c}
\gamma-\alpha \\
\beta
\end{array}\right)
$$

What one needs for (iv) is the fact that for any integer $j$,

$$
\left(\begin{array}{c}
j p^{s} \\
p^{s}
\end{array}\right) \equiv j \bmod p
$$

which follows immediately from Lucas's theorem.

We also want to point out that as with ordinary partial derivatives, the same proof as in Lemma 2.3 gives

Lemma 3.2 (Logarithmic derivative lemma). Let $f$ be an entire function in $\mathcal{E}_{m}$ and let $\gamma$ be a multi-index. Then

$$
\left|D^{\gamma} f\right|_{r} \leq \frac{|f|_{r}}{r|\gamma|}
$$

COROLlarY 3.3. Let $f$ be an entire function in $\mathcal{E}_{m}$ and let $\gamma$ be a multiindex with $|\gamma|>0$. If $f$ divides $D^{\gamma} f$, then $D^{\gamma} f \equiv 0$.

Proof. This follows from Lemma 3.2 and Corollary 2.2.

We will denote the fraction field of $\mathcal{E}_{m}$ by $\mathcal{M}_{m}$ and call it the field of meromorphic functions on $\mathbb{A}^{m}$. One sees immediately that one can use Proposition 3.1(ii) to inductively extend the Hasse derivatives to the field $\mathcal{M}_{m}$ and that the four properties of Proposition 3.1 continue to hold for functions in $\mathcal{M}_{m}$.

For each integer $k \geq 2$, let

$$
\mathcal{M}_{m}[k]=\left\{Q \in \mathcal{M}_{m}: D_{j}^{i} Q \equiv 0 \text { for all } 0<i<k \text { and } 1 \leq j \leq m\right\} .
$$

If $\mathbb{F}$ has positive characteristic $p$ and if $s$ is a positive integer, let

$$
\mathcal{E}_{m}\left[p^{s}\right]=\left\{g^{p^{s}}: g \in \mathcal{E}_{m}\right\} .
$$

Note that $\mathcal{E}_{m}\left[p^{s}\right]$ is a subring of $\mathcal{E}_{m}$ and that it consists of those elements $f$ in $\mathcal{E}_{m}$ that can be written as convergent power series in

$$
z^{p^{s}}=\left(z_{1}^{p^{s}}, \ldots, z_{m}^{p^{s}}\right) .
$$


Proposition 3.4.

(A) If $\mathbb{F}$ has characteristic 0 , then for all $k \geq 2$, we have $\mathcal{M}_{m}[k]=\mathbb{F}$.

(B) If $\mathbb{F}$ has positive characteristic $p$ and if $s$ is an integer $\geq 1$, then

(B1) $\mathcal{M}_{m}\left[p^{s-1}+1\right]=\mathcal{M}_{m}\left[p^{s}\right]$;

(B2) $\mathcal{M}_{m}\left[p^{s}\right]$ is the fraction field of $\mathcal{E}_{m}\left[p^{s}\right]$ and $D_{i}^{p^{s}}$ for $i=1, \ldots, m$ are derivations on $\mathcal{M}_{m}\left[p^{s}\right]$.

Proof. Clearly, we have (A). Proposition 3.1(iii) implies (B1). We show (B2) by induction on $s$. Let $Q$ be an element of $\mathcal{M}_{m}[2]=\mathcal{M}_{m}[p]$, which implies $\partial Q / \partial z_{i} \equiv 0$ for all $i=1, \ldots, m$. Write $Q=f / g$ with $f$ and $g$ relatively prime in $\mathcal{E}_{m}$. Suppose that $f$ is not in $\mathcal{E}_{m}[p]$. Then there is an irreducible element $P$ of $\mathcal{E}_{m}$ such that $P$ divides $f$ to a multiplicity not divisible by $p$ and such that $\partial P / \partial z_{i} \not \equiv 0$ for some $i$. This implies that $P$ divides $f$ to a higher power than it divides $\partial f / \partial z_{i}$. Since $\partial Q / \partial z_{i} \equiv 0$, this would then imply $P$ must divide $g$, contradicting the fact that $f$ and $g$ are relatively prime. Hence $f$ must have been in $\mathcal{E}_{m}[p]$. Similarly, $g$ must be in $\mathcal{E}_{m}[p]$. That $D_{i}^{p}$ is a derivation on $\mathcal{M}_{m}[p]$ then follows from Proposition 3.1(ii) or (iv). By (B1), we have

$$
\mathcal{M}_{m}\left[p^{s+1}\right]=\left\{Q \in \mathcal{M}_{m}\left[p^{s}\right]: D_{i}^{p^{s}} Q=0 \text { for all } i=1, \ldots, m\right\},
$$

and so the proof is completed by induction. Indeed, writing an element $Q$ of $\mathcal{M}_{m}\left[p^{s+1}\right] \subset \mathcal{M}_{m}\left[p^{s}\right]$ as $f / g$ with $f$ and $g$ relatively prime elements of $\mathcal{E}_{m}\left[p^{s}\right]$ and arguing as before using $D_{i}^{p^{s}}$ in place of the first partials, we see that every irreducible element $P$ of $\mathcal{E}_{m}$ that divides either $f$ or $g$ must divide them with multiplicity divisible by $p^{s+1}$, and hence $f$ and $g$ must be in $\mathcal{E}_{m}\left[p^{s+1}\right]$.

Theorem 3.5 (Hsia-Wang [18, Lem. 2]). Let $\mathbb{F}$ have characteristic zero (resp. positive characteristic $p$ ), and let $s \geq 1$ be an integer. Let $f=$ $\left(f_{0}, \ldots, f_{n-1}\right)$ be an $n$-tuple of entire functions. For a multi-index $\gamma$, let

$$
D^{\gamma} f=\left(D^{\gamma} f_{0}, \ldots, D^{\gamma} f_{n-1}\right) .
$$

Let $\gamma^{0}$ be the multi-index $(0, \ldots, 0)$. If $f_{0}, \ldots, f_{n-1}$ are linearly independent over $\mathbb{F}$ (resp. $\left.\mathcal{M}_{m}\left[p^{s}\right]\right)$, then there exist multi-indices $\gamma^{1}, \ldots, \gamma^{n-1}$ such that

$$
\left|\gamma^{i}\right| \leq\left|\gamma^{i-1}\right|+1 \quad\left(\text { resp. }\left|\gamma^{i}\right| \leq\left|\gamma^{i-1}\right|+p^{s-1}\right)
$$

and such that

$$
\operatorname{det}\left(\begin{array}{ccc}
f_{0} & \ldots & f_{n-1} \\
D^{\gamma^{1}} f_{0} & \ldots & D^{\gamma^{1}} f_{n-1} \\
D^{\gamma^{2}} f_{0} & \ldots & D^{\gamma^{2}} f_{n-1} \\
\vdots & \vdots & \vdots \\
D^{\gamma^{n-1}} f_{0} & \ldots & D^{\gamma^{n-1}} f_{n-1}
\end{array}\right) \not \equiv 0 .
$$


Remark. The determinant in Theorem 3.5 is called a generalized Wronskian. For polynomials in characteristic zero, this theorem, with a different proof, appears in [27]. In the case of complex entire functions of several variables, a similar theorem can be found in [15].

REMARK. Often (e.g. [18]), one tends to state this lemma with

$$
\left|\gamma^{i}\right| \leq i \quad\left(\operatorname{resp} .\left|\gamma^{i}\right| \leq i p^{s-1}\right),
$$

but we will want to give a lower bound on $\sum\left|\gamma^{i}\right|$ in terms of $\left|\gamma^{n-1}\right|$, and so for us the observation that

$$
\left|\gamma^{i-1}\right| \geq\left|\gamma^{i}\right|-1 \quad\left(\text { resp. }\left|\gamma^{i-1}\right| \geq\left|\gamma^{i}\right|-p^{s-1}\right)
$$

is important.

REMARK. We also remark here that Theorem 3.5 can be used to derive a positive characteristic Cartan-type second main theorem for linearly non-degenerate non-Archimedean analytic curves in projective space. For instance, the proof given in [12, Th. 5.1] goes through once a non-vanishing generalized Wronskian exists.

Proof of Theorem 3.5. We write the proof in the case of positive characteristic. The same proof works in characteristic zero by Proposition 3.4(A) if all powers of $p$ are replaced by 1 .

We proceed by induction on $n$. When $n=1$, the theorem is trivial. Now assume that the theorem is true for $n-1$. By the induction hypothesis, there exist multi-indices $\gamma^{0}, \gamma^{1}, \ldots, \gamma^{n-2}$ with

$$
\left|\gamma^{i}\right| \leq\left|\gamma^{i-1}\right|+p^{s-1}
$$

and such that the $D^{\gamma^{i}} f$ for $i=0, \ldots, n-2$ span an $n$-1-dimensional $\mathcal{M}_{m^{-}}$ vector subspace of $\mathcal{M}_{m}^{n}$. Let $k=\left|\gamma^{n-2}\right|+p^{s-1}$. Let $V$ be the $\mathcal{M}_{m}$-vector subspace of $\mathcal{M}_{m}^{n}$ spanned by $D^{\gamma} f$ for all $|\gamma| \leq k$. If the theorem were not true, then $V$ could not have dimension $n$, and so there exist $Q_{0}, \ldots, Q_{n-1}$ not all zero in $\mathcal{M}_{m}$ such that

$$
Q_{0} D^{\gamma} f_{0}+\cdots+Q_{n-1} D^{\gamma} f_{n-1} \equiv 0
$$

for every $\gamma$ with $|\gamma| \leq k$. Because the vectors

$$
\left(D^{\gamma^{0}} f_{j}, \ldots, D^{\gamma^{n-2}} f_{j}\right) \quad \text { for } j=0, \ldots, n-2
$$

are linearly independent over $\mathcal{M}_{m}$ by the induction hypothesis, we can assume $Q_{n-1} \equiv 1$, and hence we have

$$
Q_{0} D^{\gamma} f_{0}+\cdots+Q_{n-2} D^{\gamma} f_{n-2}+f_{n-1} \equiv 0 \quad \text { for all }|\gamma| \leq k .
$$


Our goal is to show that the $Q_{j}$ are in $\mathcal{M}_{m}\left[p^{s}\right]$. Note that

$$
\begin{aligned}
0 & =D_{l}^{1}\left[\sum_{j=0}^{n-2} Q_{j} D^{\gamma^{i}} f_{j}+D^{\gamma^{i}} f_{n-1}\right]=\sum_{j=0}^{n-2} D_{l}^{1} Q_{j} D^{\gamma^{i}} f_{j}+\sum_{j=0}^{n-1} Q_{j} D_{l}^{1} D^{\gamma^{i}} f_{j} \\
& =\sum_{j=0}^{n-2} D_{l}^{1} Q_{j} D^{\gamma^{i}} f_{j}
\end{aligned}
$$

for $i=0, \ldots, n-2$ and $l=1, \ldots, m$, where the last line follows from Proposition 3.1(iii) and equation (3). By the linear independence of the vectors $\left(D^{\gamma^{0}} f_{j}, \ldots, D^{\gamma^{n-2}} f_{j}\right)$, we conclude that $D_{l}^{1} Q_{j} \equiv 0$ for all $l$ and all $j=1, \ldots, n-2$. Thus, the $Q_{j}$ belong to $\mathcal{M}_{m}[2]=\mathcal{M}_{m}[p]$. Now assume the $Q_{j}$ belong to $\mathcal{M}_{m}\left[p^{t}\right]$ for some $t \geq 1$. By Proposition 3.4(B), we can apply $D_{l}^{p^{t+1}}$ as if it were a derivation to get

$$
\begin{aligned}
0 & =D_{l}^{p^{t+1}}\left[\sum_{j=0}^{n-2} Q_{j} D^{\gamma^{i}} f_{j}+D^{\gamma^{i}} f_{n-1}\right] \\
& =\sum_{j=0}^{n-2} D_{l}^{p^{t+1}} Q_{j} D^{\gamma^{i}} f_{j}+\sum_{j=0}^{n-1} Q_{j} D_{l}^{p^{t+1}} D^{\gamma^{i}} f_{j} .
\end{aligned}
$$

If $t<s$, we can use Proposition 3.1(iii), the fact that

$$
\left|\gamma^{i}\right| \leq\left|\gamma^{n-2}\right| \leq(k-1) p^{s-1}
$$

and equation (3) to conclude that the right-hand sum in (4) vanishes, and thus,

$$
0=\sum_{j=0}^{n-2} D_{l}^{p^{t+1}} Q_{j} D^{\gamma^{i}} f_{j} .
$$

Again, by linear independence, we conclude $D_{l}^{p^{t+1}} Q_{j} \equiv 0$ and so the $Q_{j}$ belong to $\mathcal{M}_{m}\left[p^{t+1}\right]$. Continuing in this manner, we find that the $Q_{j}$ are in $\mathcal{M}_{m}\left[p^{s}\right]$, contradicting the assumption that the $f_{j}$ are linearly independent over $\mathcal{M}_{m}\left[p^{s}\right]$.

Proposition 3.6. Let $f$ be an entire function in $\mathcal{E}_{m}$. Let $\gamma=\left(\gamma_{1}, \ldots, \gamma_{m}\right)$ be a multi-index. Let $P$ be an irreducible element of $\mathcal{E}_{m}$ that divides $f$ with exact multiplicity e. If $e>|\gamma|$, then $P^{e-|\gamma|}$ divides $D^{\gamma} f$. Moreover, if char $\mathbb{F}=p>0$ and if $e$ is divisible by $p^{s}>\max \left\{\gamma_{1}, \ldots, \gamma_{m}\right\}$, then $P^{e}$ divides $D^{\gamma} f$.

Proof. Because the $D_{i}$ commute, it suffices to show the proposition for $D^{\gamma}=D_{i}^{k}$.

In the special case that char $\mathbb{F}=p>0$ and $e$ is divisible by

$$
p^{s}>\max \left\{\gamma_{1}, \ldots, \gamma_{m}\right\},
$$


the fact that $P^{e}$ divides $D_{i}^{k} f$ follows easily from Proposition 3.1(ii) since for any non-negative integer $l$,

$$
D_{i}^{j} P^{l p^{s}}=0 \quad \text { for all } 0<j<p^{s}
$$

and since $p^{s}>k$ by assumption.

We show the general case by induction on $e$ and $k$. The case $e=k$ is trivial. We now suppose that the proposition holds for all $D_{i}^{j}$ with $j \leq k$ and for $e$ and then show that it also holds for $k$ and $e+1$. Suppose $f=P^{e+1} g$ with $g$ relatively prime to $P$. By Proposition 3.1(ii),

$$
D_{i}^{k} f=D_{i}^{k}\left(P \cdot P^{e} g\right)=P D_{i}^{k}\left(P^{e} g\right)+\sum_{j=1}^{k} D_{i}^{j} P D_{i}^{k-j}\left(P^{e} g\right) .
$$

If $e>k \geq k-j$, then by induction, $P^{e-(k-j)}$ divides $D_{i}^{k-j}\left(P^{e} g\right)$ and hence $P^{e-k}$ divides $D_{i}^{k-j}\left(P^{e} g\right)$ for all $j=0, \ldots, k$ and $P^{e+1-k}$ divides $D_{i}^{k-j}\left(P^{e} g\right)$ for all $j>0$.

4. Higher radicals and truncated counting functions. If $\mathbb{F}$ has characteristic zero, if $f$ is in $\mathcal{E}_{m}$, and if $l \geq 1$ is an integer, then clearly if $P$ is an irreducible element of $\mathcal{E}_{m}$ that divides $f$ with multiplicity $e$, then $P$ divides $\operatorname{gcd}\left(f, R(f)^{l}\right)$ with multiplicity $\min \{l, e\}$. Thus, in characteristic zero, we can define the lth truncated counting function by

$$
N_{f}^{(l)}(0, r)=N_{\operatorname{gcd}\left(f, R(f)^{l}\right)}(0, r) .
$$

We saw at the end of Section 2 that in positive characteristic $p$, the radical $R(f)$ does not contain those irreducible factors of $f$ that divide $f$ with multiplicity divisible by $p$. Although that was exactly what was appropriate in Theorem 2.6, when we consider

$$
f_{n}=f_{0}+\cdots+f_{n-1}
$$

with $n>2$, we will not be able to ignore all irreducible factors with multiplicity divisible by $p$. Thus, we want to define truncated counting functions in positive characteristic that include all irreducible factors.

For the rest of this section, let $\mathbb{F}$ have positive characteristic $p$. We will use the following proposition to inductively define higher $p^{s}$-radicals for integers $s \geq 1$.

Proposition 4.1. Let $f$ be an entire function in $\mathcal{E}_{m}$ and let $s \geq 1$ be an integer. Assume that we have defined a $p^{s-1}$-radical $R_{p^{s-1}}(f)$ that has the property that $R_{p^{s-1}}(f)$ is square-free and has the property that an irreducible element $P$ of $\mathcal{E}_{m}$ divides $R_{p^{s-1}}(f)$ if and only if $P$ divides $f$ with multiplicity not divisible by $p^{s}$. Let

$$
\bar{f}=\frac{f}{\operatorname{gcd}\left(f, R_{p^{s-1}}(f)^{p^{s}}\right)} .
$$


For $i=1, \ldots, m$, let $g_{i}=\operatorname{gcd}\left(\bar{f}, D_{i}^{p^{s}} \bar{f}\right)$, let $h_{i}=\bar{f} / g_{i}$, and let $H$ be the least common multiple of the $h_{i}$. Let

$$
G=\frac{H}{\operatorname{gcd}\left(H, R_{p^{s-1}}(H)^{p^{s}-1}\right)} .
$$

Then:

(i) if $P$ is an irreducible element of $\mathcal{E}_{m}$ that divides $G$, then it divides $G$ with multiplicity exactly $p^{s}$;

(ii) if $P$ is an irreducible element of $\mathcal{E}_{m}$, then $P$ divides $G$ if and only if $P$ divides $f$ with multiplicity a multiple of $p^{s}$ but not a multiple of $p^{s+1}$.

It follows that $G$ is a $p^{s}$ th power, so we can let $R$ be a $p^{s}$ th root of $G$ and let $R_{p^{s}}(f)$ be the least common multiple of $R_{p^{s-1}}(f)$ and $R$.

Proof. Our induction begins with the radical as defined in Section 2, so we let $R_{p^{0}}(f)=R(f)$. To show the inductive step, let $P$ be an irreducible element of $\mathcal{E}_{m}$ that divides $\bar{f}$. Note that if $P$ divides $\bar{f}$, then it divides it with multiplicity at least $p^{s}$. Write

$$
\bar{f}=P^{j p^{s}+e} \tilde{f},
$$

where $j \geq 1$ and $0 \leq e<p^{s}$ are integers and $\tilde{f}$ is relatively prime to $P$. Then, by Proposition 3.1(ii) and (iv) and Proposition 3.4(B), for $i=1, \ldots, m$,

$$
D_{i}^{p^{s}} \bar{f}=D_{i}^{p^{s}}\left[P^{j p^{s}} P^{e} \widetilde{f}\right]=P^{e} \tilde{f}\left(D_{i}\left[P^{j}\right]\right)^{p^{s}}+P^{j p^{s}} D_{i}^{p^{s}}\left[P^{e} \widetilde{f}\right] .
$$

We first consider the case that $j$ is not divisible by $p$. Because $P$ is irreducible and hence not a $p$ th power, there exists an $i$ such that $D_{i} P \not \equiv 0$. Because

$$
D_{i}\left(P^{j}\right)=j P^{j-1} D_{i} P,
$$

we see from the assumption that $j$ is not divisible by $p$, that $P$ divides $D_{i}^{p^{s}} f$ with exact multiplicity $(j-1) p^{s}+e$, and so by Proposition 3.6, $P$ divides $H$ with exact multiplicity $p^{s}$.

In case $j$ is divisible by $p$, we see from equation (5) that $P$ divides $H$ with multiplicity at most $e<p^{s}$. Thus, $P$ does not divide $R$.

We now show the existence of the square-free part of an entire function, which is square-free and contains all the irreducible factors dividing the function.

THEOREM 4.2. Let $f$ be an entire function in $\mathcal{E}_{m}$. There exists an entire function $S(f)$ in $\mathcal{E}_{m}$ such that $S(f)$ is square-free and such that an irreducible element $P$ in $\mathcal{E}_{m}$ divides $S(f)$ if and only if it divides $f$. 
We will call $S(f)$ the square-free part of $f$. We define the lth truncated counting function by

$$
N_{f}^{(l)}(0, r)=N_{\operatorname{gcd}\left(f, S(f)^{l}\right)}(0, r) .
$$

As in characteristic zero, the $l$ th truncated counting function truncates to $l$ all multiplicities higher than $l$.

Proof of Theorem 4.2. The proof is similar to the proof of the existence of greatest common divisors given in [11]. The case that $f$ is identically zero is trivial, so assume that $f$ is not identically zero. For $i=1,2, \ldots$, let $r_{i}$ be an increasing sequence of elements of $\left|\mathbb{F}^{\times}\right|$such that $r_{i} \rightarrow \infty$. Consider $f$ as an element of the ring $\mathcal{A}_{m}\left(r_{i}\right)$ of analytic functions on $\mathbf{B}^{m}\left(r_{i}\right)$, the closed ball of radius $r_{i}$. Let $z_{0}$ be a point in $\mathbf{B}^{m}\left(r_{1}\right)$ such that $f\left(z_{0}\right) \neq 0$. Let $R_{p^{s}}(f)$ be the higher radicals of $f$ defined as in Proposition 4.1 normalized so that for each $s$, we have $R_{p^{s}}(f)\left(z_{0}\right)=1$. Because the ring $\mathcal{A}_{m}\left(r_{i}\right)$ is factorial $\left[6, \S 5.2 .6\right.$, Th. 1], only finitely many of the irreducible elements in $\mathcal{E}_{m}$ that divide $f$ are non-units in $\mathcal{A}_{m}\left(r_{i}\right)$. Each of these divides $f$ to some finite multiplicity, and so there exists some $s_{i}$ such that every irreducible element in $\mathcal{E}_{m}$ that divides $f$ and is not a unit in $\mathcal{A}_{m}\left(r_{i}\right)$ also divides $R_{p^{s}}(f)$ for all $s \geq s_{i}$. This means that for $s, t \geq s_{i}, R_{p^{s}}(f)$ and $R_{p^{t}}(f)$ differ by a unit in $\mathcal{A}_{m}\left(r_{n}\right)$.

Let $u_{i, i+1}$ be the unit in $\mathcal{A}^{m}\left(r_{i}\right)$ such that

$$
R_{p^{s_{i}}}(f)=u_{i, i+1} R_{p^{s_{i+1}}}(f),
$$

and note that $u_{i, i+1}\left(z_{0}\right)=1$. Then, writing $u_{i, i+1}$ as a power series about $z_{0}$, we have

$$
u_{i, i+1}(z)=1+\sum_{|\gamma| \geq 1} a_{\gamma}\left(z-z_{0}\right)^{\gamma} \quad \text { with }\left|a_{\gamma}\right| r_{i}^{|\gamma|}<1 \text { for all }|\gamma| \geq 1 .
$$

Thus, for $j>i$,

$$
\left|u_{j, j+1}-1\right|_{r_{i}}<\frac{r_{i}}{r_{j}}
$$

Since $r_{i} / r_{j} \rightarrow 0$ as $j \rightarrow \infty$ with $i$ fixed, we can define units $v_{i}$ in $\mathcal{A}_{m}\left(r_{i}\right)$ by

$$
v_{i}=\prod_{j=i}^{\infty} u_{j, j+1} .
$$

For $j>i$, we have

$$
\begin{aligned}
R_{p^{s_{j}}}(f) v_{i} & =R_{p^{s_{j}}}(f) \prod_{k=i}^{\infty} u_{k, k+1}=R_{p^{s_{j}}}(f)\left(\prod_{k=i}^{j-1} u_{k, k+1}\right)\left(\prod_{k=j}^{\infty} u_{k, k+1}\right) \\
& =R_{p^{s_{i}}}(f) v_{j} .
\end{aligned}
$$


This precisely means that $R_{p^{s^{i}}}(f) v_{i}^{-1}$ converges to an entire function $F$ as $i \rightarrow \infty$ because the difference between $R_{p^{s^{i}}}(f) v_{i}^{-1}$ and $R_{p^{s}}(f) v_{j}^{-1}$ is identically zero on $\mathbf{B}^{m}\left(r_{i}\right)$. Note also that $v_{i} F=R_{p^{s_{i}}}(f)$ in $\mathcal{A}_{m}\left(r_{i}\right)$.

We claim that $F$ is square free and that an irreducible element $P$ of $\mathcal{E}_{m}$ divides $F$ if and only if it divides $f$.

To show that $F$ is square free, suppose that $P$ is an irreducible element of $\mathcal{E}_{m}$ such that $P^{2}$ divides $F$. This means $P^{2}$ divides $R_{p^{s}}(f)$ in $\mathcal{A}_{m}\left(r_{i}\right)$. For $i$ sufficiently large, $P$ is not a unit in $\mathcal{A}_{m}\left(r_{i}\right)$, and so it would be the case that $R_{p^{s_{i}}}(f)$ is not square free in $\mathcal{A}_{m}\left(r_{i}\right)$. However, the proof of Proposition 4.1 works equally well in the ring $\mathcal{A}_{m}\left(r_{i}\right)$, and thus $R_{p^{s_{i}}}(f)$ is also square free in $\mathcal{A}_{m}\left(r_{i}\right)$.

Finally, let $P$ be an irreducible element of $\mathcal{E}_{m}$. Suppose $P$ divides $f$. Then $P$ divides $R_{p^{s}}(f)$ for all $i$ sufficiently large. In other words, there exist analytic functions $h_{i}$ in $\mathcal{A}_{m}\left(r_{i}\right)$ such that

$$
P h_{i}=R_{p^{s_{i}}}(f) .
$$

Because $P h_{i} v_{i}^{-1}$ converges to $F$ as $i \rightarrow \infty$, for $j>i$, we have

$$
P\left(h_{i} v_{i}^{-1}-h_{j} v_{j}^{-1}\right)=0 \quad \text { in } \mathcal{A}_{m}\left(r_{i}\right) .
$$

Thus $h_{i} v_{i}^{-1}$ converges to an entire function $H$ such that $P H=F$, and so $P$ divides $F$. For the other direction, suppose that $P$ divides $F$. Then $P$ divides $R_{p^{s_{i}}}(f)$ in $\mathcal{A}_{m}\left(r_{i}\right)$, and so again noticing that the proof of Proposition 4.1 also works for $\mathcal{A}_{m}\left(r_{i}\right)$, we deduce that $P$ divides $f$ in $\mathcal{A}_{m}\left(r_{i}\right)$. In other words, there exist analytic functions $g_{i}$ in $\mathcal{A}_{m}\left(r_{i}\right)$ such that $P g_{i}=f$. This implies that the $g_{i}$ converge to an analytic function $G$ such that $P G=f$, and hence $P$ divides $f$ in $\mathcal{E}_{m}$.

5. Linear algebra. Let $V$ be a vector space over a field $\mathbb{E}$. Let $v_{0}, \ldots, v_{n}$ be $n+1$ linearly dependent vectors in $V$. Call an index set $I \subset\{0, \ldots, n\}$ minimal if the set of vectors $\left\{v_{i}: i \in I\right\}$ is linearly dependent, but such that for every proper subset $I^{\prime} \subsetneq I$, the sets of vectors $\left\{v_{i}: i \in I^{\prime}\right\}$ are linearly independent.

Lemma 5.1 (Brownawell-Masser). Let $v_{0}, \ldots, v_{n}$ be $n+1$ vectors in a vector space $V$ over a field $\mathbb{E}$ such that $\sum v_{i}=0$. Assume that no proper subsum vanishes, i.e.,

$$
\sum_{i \in I} v_{i} \neq 0 \quad \text { for all } I \subsetneq\{1, \ldots, n\} .
$$

Then there exists an integer $u \geq 1$, a partition

$$
\{0, \ldots, n\}=I_{0} \cup \cdots \cup I_{u-1},
$$


and non-empty subsets

$$
J_{l} \subset \bigcup_{j=1}^{l} I_{j}, \quad l=0, \ldots, u-2,
$$

such that $I_{0}$ and $I_{j} \cup J_{j-1}$ for $j=1, \ldots, u-1$ are minimal.

Proof. If $\{0, \ldots, n\}$ is minimal, set $I_{0}=\{0, \ldots, n\}$. If $\{0, \ldots, n\}$ is not minimal, see [10, Lem. 6].

In positive characteristic $p$, we want to apply Theorem 3.5 to entire functions linearly independent over $\mathbb{F}$, so we complete this section by proving that if a collection of functions are linearly independent over $\mathbb{F}$, then they are also linearly independent over $\mathcal{M}_{m}\left[p^{s}\right]$ for some integer $s \geq 1$.

LEMMA 5.2. Let $f_{1}, \ldots, f_{n}$ be meromorphic functions in $\mathcal{M}_{m}$ linearly independent over $\mathbb{F}$, with char $\mathbb{F}=p>0$. Then there exists an integer $s \geq 1$ such that $f_{1}, \ldots, f_{n}$ are linearly independent over $\mathcal{M}_{m}\left[p^{s}\right]$.

Proof. Suppose the lemma is not true. Then, $f_{1}, \ldots, f_{n}$ are linearly dependent over $\mathcal{M}_{m}\left[p^{s}\right]$ for every $s \geq 1$. For each $s \geq 1$, let $I_{s} \subset\{1, \ldots, n\}$ be minimal. Note that each $I_{s}$ contains at least two indices, otherwise one of the functions $f_{j}$ would be identically zero, and hence the $f_{j}$ could not be linearly independent over $\mathbb{F}$. Because there are only finitely many possible subsets $I_{s}$, we may assume without loss of generality that $I_{s}=\{1, \ldots, t\}$ for infinitely many $s$. Thus, for infinitely many $s$, the functions $f_{2}, \ldots, f_{t}$ are linearly independent over $\mathcal{M}_{m}\left[p^{s}\right]$ and $f_{1}$ is in the $\mathcal{M}_{m}\left[p^{s}\right]$-linear span of $f_{2}, \ldots, f_{t}$. In other words, for infinitely many $s$ there exist unique $Q_{s, 2}, \ldots, Q_{s, t}$ in $\mathcal{M}_{m}\left[p^{s}\right]$ such that

$$
f_{1}=Q_{s, 2} f_{2}+\cdots+Q_{s, t} f_{t} .
$$

On the other hand, $\mathcal{M}_{m}\left[p^{s^{\prime}}\right] \subset \mathcal{M}_{m}\left[p^{s}\right]$ if $s^{\prime} \geq s$, so by the linear independence of $f_{2}, \ldots, f_{t}$, the $Q_{s, j}$ do not depend on $s$. Hence, $Q_{s, j}$ is in $\mathcal{M}_{m}\left[p^{s}\right]$ for infinitely many $s$, and must therefore be in $\mathbb{F}$. This contradicts the linear independence of the $f_{j}$ over $\mathbb{F}$.

If $\mathbb{F}$ has positive characteristic $p$, then we define the index of independence of a collection $\mathcal{F}$ of entire or meromorphic functions to be the smallest integer $s$ such that any subset of functions in $\mathcal{F}$ linearly independent over $\mathbb{F}$ remains linearly independent over $\mathcal{M}_{m}\left[p^{s}\right]$, provided such an integer exists. Lemma 5.2 shows that such an integer always exists if $\mathcal{F}$ is finite.

6. ABC theorems. In the three-function $\mathrm{ABC}$ theorem, one begins with $f_{2}=f_{0}+f_{1}$ with the $f_{i}$ relatively prime. Note that here $\operatorname{gcd}\left(f_{0}, f_{1}, f_{2}\right)=1$ implies that the $f_{j}$ are also pairwise relatively prime because, by the linear dependence, if two of the functions have a common factor, it divides the third 
as well. To generalize to $n+1$ functions, one obviously wants to consider

$$
f_{n}=f_{0}+\cdots+f_{n-1},
$$

or more symmetrically,

$$
0=f_{0}+\cdots+f_{n} .
$$

Some work on such generalizations, e.g. [29], assumes the rather strong hypothesis that the $f_{j}$ are pairwise relatively prime. Other work, e.g. [10], assumes $\operatorname{gcd}\left(f_{0}, \ldots, f_{n}\right)=1$ and that

$$
\sum_{i \in I} f_{i} \neq 0 \quad \text { for each proper index subset } I \subsetneq\{0, \ldots, n\} .
$$

This hypothesis is referred to as "no vanishing subsums". The recent work of De Bondt [5] generalizes these two hypotheses to the following: Suppose $0=f_{0}+\cdots+f_{n}$ and assume that for each index set $I \subseteq\{0, \ldots, n\}$,

$$
\text { if } \sum_{i \in I} f_{i}=0, \quad \text { then } \operatorname{gcd}\left(\left\{f_{i}: i \in I\right\}\right)=1 .
$$

In the three-function $\mathrm{ABC}$ theorem, the right-hand side of the $\mathrm{ABC}$ inequality involves the radical $R\left(f_{0} f_{1} f_{2}\right)$ of the product. But because the functions are pairwise relatively prime, this is the same as the product of the radicals: $R\left(f_{0}\right) R\left(f_{1}\right) R\left(f_{2}\right)$. When one begins with $n+1$ functions that are not necessarily pairwise relatively prime, then the square free part of the product, $S\left(f_{0} \cdots f_{n}\right)$, will not in general be the same as the product of the square free parts, $S\left(f_{0}\right) \cdots S\left(f_{n}\right)$. Again, we follow De Bondt's lead by presenting generalized $\mathrm{ABC}$ inequalities of both types.

The following two theorems are our generalized ABC theorems for nonArchimedean entire functions of several variables.

TheOREM 6.1 (Generalized ABC theorem (first version)). Let $f_{0}, \ldots, f_{n}$ be $n+1 \geq 3$ entire functions in $\mathcal{E}_{m}$, not all of which are constant and none of which is identically zero. Assume

$$
0=f_{0}+\cdots+f_{n}
$$

and assume that for each index set $I \subseteq\{0, \ldots, n\}$,

$$
\text { if } \sum_{i \in I} f_{i}=0 \text { then } \operatorname{gcd}\left(\left\{f_{i}: i \in I\right\}\right)=1 .
$$

Let $2 \leq d \leq n$ be the dimension of the $\mathbb{F}$-vector space spanned by the $f_{i}$. If $\mathbb{F}$ has characteristic zero, let $c=1$, and if $\mathbb{F}$ has positive characteristic $p$, let $c=p^{s-1}$, where $s$ is the index of independence for the $f_{i}$. Then there exist integers $a$ and $b$ with

$$
1 \leq a \leq c(d-1) \quad \text { and } \quad b \geq a\left\lceil\frac{a}{c}\right\rceil-\frac{\left\lceil\frac{a}{c}\right\rceil\left(\left\lceil\frac{a}{c}\right\rceil-1\right)}{2} c \geq a
$$


such that for $r \geq 1$,

$$
\max _{0 \leq j \leq n} \log \left|f_{j}\right|_{r} \leq \sum_{j=0}^{n} N_{f_{j}}^{(a)}(0, r)-b \log r+O(1) .
$$

Moreover, if char $\mathbb{F}=p>0$ then there further exists a non-negative integer $\sigma$ with $p^{\sigma} \leq$ a such that for $r \geq 1$,

$$
\max _{0 \leq j \leq n} \log \left|f_{j}\right|_{r} \leq \sum_{j=0}^{n} N_{G_{j}}(0, r)-b \log r+O(1),
$$

where $G_{j}=\operatorname{gcd}\left(f_{j}, R_{p^{\sigma}}\left(f_{j}\right)^{a}\right)$.

TheOREM 6.2 (Generalized ABC theorem (second version)). Let $f_{0}, \ldots$ $\ldots, f_{n}$ be $n+1 \geq 3$ entire functions in $\mathcal{E}_{m}$, not all of which are constant and none of which is identically zero. Assume

$$
0=f_{0}+\cdots+f_{n} .
$$

Let $2 \leq d \leq n$ be the dimension of the $\mathbb{F}$-vector space spanned by the $f_{i}$. Let $2 \leq k \leq n$ and assume that

$$
\operatorname{gcd}\left(f_{i_{1}}, \ldots, f_{i_{k}}\right)=1 \quad \text { for any } 0 \leq i_{1}<\cdots<i_{k} \leq n .
$$

Let $\bar{k}=\min \{k, d\}$. If $\bar{k}>2$, further assume

$$
\sum_{i \in I} f_{i} \neq 0 \quad \text { for each } I \subsetneq\{0, \ldots, n\} .
$$

If $\mathbb{F}$ has characteristic zero, let $c=1$, and if $\mathbb{F}$ has positive characteristic $p$, let $c=p^{s-1}$, where $s$ is the index of independence for the $f_{i}$. Then there exist integers $\bar{a}$ and $b$ with

$$
1 \leq \bar{a} \leq c \sum_{i=1}^{\bar{k}-1}(d-i) \quad \text { and } \quad \bar{a} \leq b
$$

such that for $r \geq 1$,

$$
\max _{0 \leq j \leq n} \log \left|f_{j}\right|_{r} \leq N_{F}^{(\bar{a})}(0, r)-b \log r+O(1)
$$

where $F=f_{0} \cdots f_{n}$

We remark that given explicit $f_{i}$, the constants $a, b, \sigma$, and $\bar{a}$ in Theorems 6.1 and 6.2 can be determined explicitly in terms of non-vanishing generalized Wronskians of subsets of the $f_{i}$, as will be evident from the proof; see (16), (17), (18), and (20).

Trivial examples in positive characteristic show that the dependence on the index of independence $s$ cannot be removed.

In characteristic zero and one variable, Theorems 6.1 and 6.2 are due to $\mathrm{Hu}$ and Yang [20]. Below, we will give their proof, which in turn closely follows Brownawell and Masser [10], and simply observe that it works, given 
the proper set-up, just as well for several variables and in positive characteristic. That it is natural to express the upper bounds on $a$ and $\bar{a}$ in terms of $d$ is an observation of Zannier [35]. For polynomials of several variables, De Bondt [5] gave an alternative Wronskian-based proof that first reduces to the case of one-variable polynomials by generic specialization but then proves the one-variable case by introducing extra variables to force linear independence, thereby avoiding Lemma 5.1.

Before giving the proof of Theorems 6.1 and 6.2, we discuss how to derive from it various other existing results in the literature. First observe that in characteristic zero, $b \geq a(a+1) / 2$ and so (8) implies

$$
\max _{0 \leq j \leq n} \log \left|f_{j}\right|_{r} \leq \sum_{j=0}^{n} N_{f_{j}}^{(a)}(0, r)-\frac{a(a+1)}{2} \log r+O(1),
$$

which specializing to complex polynomials gives us

Corollary 6.3 ([5, Th. 2.1(4)]). Let $f_{0}, \ldots, f_{n}$ be polynomials of several complex variables satisfying the hypothesis of the theorem. Then

$$
\max \operatorname{deg} f_{j} \leq \sum_{j=0}^{n} r_{a}\left(f_{j}\right)-\frac{a(a+1)}{2},
$$

where $r_{a}\left(f_{j}\right)=\operatorname{deg} \operatorname{gcd}\left(f_{j}, R\left(f_{j}\right)^{a}\right)$ and $a$ is as in the theorem.

Incorporating an idea of Bayat and Teimoori [4] as in De Bondt [5], we get

COROllary 6.4. Assume $\mathbb{F}$ has characteristic zero, let $f_{0}+\cdots+f_{n}=0$ be as in Theorem 6.1 and let $d$ be the dimension of the $\mathbb{F}$-vector space spanned by the $f_{j}$. Let $C$ be the number of $f_{j}$ which are constant functions. For any $A$ with $d \leq A \leq n-C$,

$$
\max _{0 \leq j \leq n} \log \left|f_{j}\right|_{r} \leq A\left(\sum_{j=0}^{n} N_{f_{j}}^{(1)}(0, r)-\frac{A+1}{2} \log r\right)+O(1)
$$

for $r \geq 1$.

De Bondt gives examples that show that $A$ cannot be made smaller than $d$ in Corollary 6.4.

In the case of polynomials, Corollary 6.4 gives [5, Th. 2.1(5)], which implies [4, Th. 5] as explained in [5]. As remarked by De Bondt, the proof of [4, Th. 5] given by Bayat and Teimoori in [4] is not correct for polynomials of several variables because their Lemma 4 is easily seen to be false for several-variable polynomials. However, arguing as in [5], their Theorem 5 is correct, even for several variables. Of course, this also recovers the result of Shapiro and Sparer [29]. 
Proof of Corollary 6.4. Clearly (by Proposition 2.7),

$$
N_{f_{j}}^{(a)}(0, r) \leq a N_{f_{j}}^{(1)}(0, r),
$$

so from (8), we get

$$
\max _{0 \leq j \leq n} \log \left|f_{j}\right|_{r} \leq a\left(\sum_{j=0}^{n} N_{f_{j}}^{(1)}(0, r)-\frac{a+1}{2} \log r\right)+O(1)
$$

for $r \geq 1$. The observation of Bayat and Teimoori is that the expression on the right is quadratic in $a$ and hence increasing in $a$ provided

$$
a+\frac{1}{2} \leq \frac{1}{\log r} \sum_{j=0}^{n} N_{f_{j}}^{(1)}(0, r) .
$$

Because

$$
N_{f_{j}}^{(1)}(0, r) \geq \log r+O(1)
$$

for any non-constant $f_{j}$ and because there are $n+1-C$ non-constant $f_{j}$, we have

$$
\sum_{j=0}^{n} N_{f_{j}}^{(1)}(0, r) \geq(n+1-C) \log r+O(1) \geq(n-C) \log r
$$

for $r$ sufficiently large, and so we can increase $a$ up to $n-C$ and the inequality will hold for all sufficiently large $r$. We can then adjust the $O(1)$ term to make the inequality hold for $r \geq 1$.

We now digress a little bit to discuss one slightly subtle difference between entire functions and polynomials. The astute reader will notice that we assumed no vanishing subsums, i.e. (11), in Theorem 6.2, whereas De Bondt assumed the weaker hypothesis (7) in both versions of his ABC theorems. In the case of complex polynomials, the inequality in De Bondt's work that corresponds to our inequality (12) is

$$
\max \operatorname{deg} f_{j} \leq \operatorname{deg} \operatorname{gcd}\left(F, R(F)^{\bar{a}}\right)-b \leq \operatorname{deg} \operatorname{gcd}\left(F, R(F)^{\bar{a}}\right)-\bar{a},
$$

and this holds even if the hypothesis (11) of no vanishing subsums is weakened to (7). The reason for this is that in the case of polynomials, one of the polynomials $f_{j_{0}}$ has maximal degree. Thus, one need only consider a minimal index set $I$ such that $j_{0}$ is contained in $I$ and such that

$$
\sum_{j \in I} f_{j}=0
$$

However, in the case of entire functions, it need not be the case that there is a fixed index $j_{0}$ such that for all $r$ sufficiently large

$$
\left|f_{j_{0}}\right|_{r}=\max _{0 \leq j \leq n}\left|f_{j}\right|_{r}
$$


Thus, if one replaces the hypothesis (11) in Theorem 6.2 with the weaker hypothesis (7), it follows from (12) and the fact that for a positive integer $l$, for $r \geq 1$ and $F$ and $G$ entire functions we have

$$
\max \left\{N_{F}^{(l)}(0, r), N_{G}^{(l)}(0, r)\right\} \leq N_{F G}^{(l)}(0, r),
$$

that for each index $j$ in $\{0, \ldots, n\}$, there exist integers $\bar{a}_{j}$ and $b_{j}$ with

$$
1 \leq \bar{a}_{j} \leq c \sum_{i=1}^{k}(d-i) \quad \text { and } \quad \bar{a}_{j} \leq b_{j}
$$

such that for $r \geq 1$,

$$
\log \left|f_{j}\right|_{r} \leq N_{F}^{\left(\bar{a}_{j}\right)}(0, r)-b_{j} \log r+O(1),
$$

from which it follows that

$$
\max _{0 \leq j \leq n} \log \left|f_{j}\right|_{r} \leq N_{F}^{\left(\max \bar{a}_{j}\right)}(0, r)-\left(\min b_{j}\right) \log r+O(1) .
$$

Of course, it need not be that $\min b_{j} \geq \max \bar{a}_{j}$, and thus when subsums of the $f_{j}$ may vanish, it is not clear for entire functions whether one can choose the same constant at which multiplicities are truncated when counting the zeros of $F$ as the coefficient in front of $-\log r$. Whether that can be done is a somewhat interesting question, because if it can be done, then the proof cannot be a straightforward generalization of the existing polynomial proof. If it cannot be done, then this would be an example where a polynomial inequality does not completely generalize to an analogous inequality for entire functions.

COROLlary 6.5. With hypotheses and notation as in Theorem 6.2, we have, for $r \geq 1$ and any

$$
\bar{A} \geq c \sum_{i=1}^{\bar{k}-1}(d-i)
$$

the inequality

$$
\max _{0 \leq j \leq n} \log \left|f_{j}\right|_{r} \leq \bar{A}\left(N_{F}^{(1)}(0, r)-\log r\right)+O(1) .
$$

Moreover, the above inequality remains valid if the hypothesis (11) is weakened to hypothesis (7).

Remark. The $O(1)$ term may depend on $\bar{A}$.

Proof of Corollary 6.5. Under the hypothesis (11), we have

$$
\max _{0 \leq j \leq n} \log \left|f_{j}\right|_{r} \leq N_{F}^{(\bar{a})}(0, r)-\bar{a} \log r+O(1),
$$

which follows immediately from (12) because $\bar{a} \leq b$. Because

$$
N_{F}^{(\bar{a})}(0, r) \leq \bar{a} N_{F}^{(1)}(0, r)
$$


and because

$$
N_{F}^{(1)}(0, r)-\log r
$$

is bounded below for $r \geq 1$ since $F$ is non-constant, we have

$$
N_{F}^{(\bar{a})}(0, r)-\bar{a} \log r \leq \bar{a}\left(N_{F}^{(1)}(0, r)-\log r\right) \leq \bar{A}\left(N_{F}^{(1)}(0, r)-\log r\right)+O(1),
$$

which gives the corollary when there are no vanishing subsums. However, even if there are vanishing subsums, the functions can be grouped into vanishing subsums

$$
\sum_{i \in I} f_{i}=0
$$

with no vanishing sub-subsums. Any vanishing subsum consisting of all constants can be thrown out. Letting

$$
F_{I}=\prod_{i \in I} f_{i}
$$

we have

$$
\max _{j \in I} \log \left|f_{j}\right|_{r} \leq \bar{A}\left(N_{F_{I}}^{(1)}(0, r)-\log r\right)+O(1) .
$$

Because $\bar{A}$ was chosen independent of $I$ and because

$$
\max _{I} N_{F_{I}}^{(1)}(0, r) \leq N_{F}^{(1)}(0, r) \quad \text { for } r \geq 1,
$$

the corollary follows in general.

If $\mathbb{F}$ has characteristic zero and in the case of polynomials when $k=n$, Corollary 6.5 is [5, Th. 2.2(7)]. When $k=3$, then we can take $\bar{A}=2 n-3$ and so we recover the main result of Quang and Tuan in [26]:

$$
\max _{0 \leq j \leq n} \operatorname{deg} f_{j} \leq(2 n-3)[\operatorname{deg} R(F)-1]
$$

if $\operatorname{gcd}\left(f_{i_{1}}, f_{i_{2}}, f_{i_{3}}\right)=1$ for all triples of indices $i_{1}<i_{2}<i_{3}$. Note that Quang and Tuan neglected the necessary hypothesis that the functions in any vanishing subsum be relatively prime, i.e., hypothesis (7). We also remark that Browkin and Brzeziński [9] conjectured that (13) remains true for one-variable polynomials in characteristic zero if the gcd hypothesis is relaxed to $\operatorname{gcd}\left(f_{0}, \ldots, f_{n}\right)=1$ and no vanishing subsums. This conjecture seems to be out of reach of the current Wronskian-based proofs.

Fundamental to the proof of Theorems 6.1 and 6.2 are the following lemmas about generalized Wronskians.

Lemma 6.6. Let $f_{0}, \ldots, f_{n-1}$ be entire functions in $\mathcal{E}_{m}$. Let

$$
\gamma^{1}=\left(\gamma_{1}^{1}, \ldots, \gamma_{m}^{1}\right), \quad \ldots, \quad \gamma^{n-1}=\left(\gamma_{1}^{n-1}, \ldots, \gamma_{m}^{n-1}\right)
$$

be multi-indices with $\left|\gamma^{1}\right| \leq \cdots \leq\left|\gamma^{n-1}\right|$ such that the associated generalized Wronskian $W$ does not vanish identically. Let $\gamma^{0}=(0, \ldots, 0)$. If $P$ is an irreducible element which divides $f_{i}$ with multiplicity $e>\left|\gamma^{n-1}\right|$, then $P$ 
divides $W$ with multiplicity at least $e-\left|\gamma^{n-1}\right|$. Moreover, if $\operatorname{char} \mathbb{F}=p>0$ and if $p^{t}$ divides $e$ and

$$
p^{t}>\max \left\{\gamma_{i}^{j}: 1 \leq j \leq n-1 \text { and } 1 \leq i \leq m\right\},
$$

then $P^{e}$ divides $W$.

REMARK. Note that $\max \left\{\gamma_{i}^{j}: 1 \leq j \leq n-1\right.$ and $\left.1 \leq i \leq m\right\} \leq\left|\gamma^{n-1}\right|$.

Proof of Lemma 6.6. This follows immediately from Proposition 3.6.

Lemma 6.7. Let $f_{0}, \ldots, f_{n-1}$ be entire functions in $\mathcal{E}_{m}$. Let $\gamma^{1}, \ldots, \gamma^{n-1}$ be multi-indices with $\left|\gamma^{1}\right| \leq \cdots \leq\left|\gamma^{n-1}\right|$ such that the associated generalized Wronskian $W$ does not vanish identically. Let $\gamma^{0}=(0, \ldots, 0)$ and let $F=f_{0} \cdots f_{n-1}$. Let $k \geq 2$ be the smallest integer such that for any $k$ distinct indices $i_{1}, \ldots, i_{k}$ in $\{0, \ldots, n-1\}$ one has $\operatorname{gcd}\left(f_{i_{1}}, \ldots, f_{i_{k}}\right)=1$, or if no such $k$ exists, let $k=n+1$. Let

$$
\ell=\sum_{i=1}^{k-1}\left|\gamma^{n-i}\right|
$$

If $P$ is an irreducible element which divides $F$ with multiplicity $e>\ell$, then $P$ divides $W$ with multiplicity at least $e-\ell$.

REMARK. Note that in positive characteristic $p$, even if $P$ divides $F$ with multiplicity a large multiple of $p$, this does not necessarily mean that $P$ divides $W$ with multiplicity divisible by $p$. This is because the powers of $P$ may be split among the different $f_{i}$, so $P$ need not divide any of the $f_{i}$ with multiplicity divisible by $p$.

Proof of Lemma 6.7. By the hypothesis of the lemma, we may assume without loss of generality, by re-ordering the indices if necessary, that $P$ does not divide $f_{j}$ for $j \geq k-1$. Let $e_{i} \geq 0$ for $i=0, \ldots, k-2$ be the multiplicities with which $P$ divides the $f_{i}$, and assume $e_{0} \geq \cdots \geq e_{k-2}$. Then, by Proposition 3.6, $P$ divides $W$ with multiplicity at least

$$
\sum_{i=0}^{k-2} \max \left\{0, e_{i}-\left|\gamma^{n-i-1}\right|\right\} \geq \sum_{i=0}^{k-2}\left(e_{i}-\left|\gamma^{n-i-1}\right|\right)=e-\ell .
$$

Proof of Theorems 6.1 and 6.2. If $\mathbb{F}$ has positive characteristic, let $G_{i}$ be as in the statement of Theorem 6.1. If $\mathbb{F}$ has characteristic zero, let

$$
G_{i}=\operatorname{gcd}\left(f_{i}, S\left(f_{i}\right)^{a}\right) .
$$

Then $G_{i}$ divides $\operatorname{gcd}\left(f_{i}, S\left(f_{i}\right)^{a}\right)$, and thus

$$
N_{G_{i}}(0, r) \leq N_{f_{i}}^{(a)}(0, r)
$$

for $r \geq 1$ by Proposition 2.7 and the definition of truncated counting functions. Therefore, inequality (8) follows from inequality (9). Hence, to prove 
Theorem 6.1, it suffices to prove (9), where in characteristic zero we interpret (9) with $G_{i}$ as defined here in the proof.

We next observe that it suffices to prove Theorem 6.1 assuming there are no vanishing subsums. Indeed, if there are vanishing subsums, simply group the $f_{i}$ into vanishing subsums with no vanishing sub-subsums, and note that these subsums still satisfy all the hypotheses of the theorem. Summing (9) over each minimal vanishing subsum clearly results in (9) for the general case.

Note also that if $\bar{k}=2$ in Theorem 6.2 , then the $f_{j}$ are pairwise relatively prime, in which case

$$
N_{F}^{(\ell)}(0, r)=\sum_{j=0}^{n} N_{f_{j}}^{(\ell)}(0, r),
$$

and so Theorem 6.2 follows from Theorem 6.1. Thus, we henceforth assume (11) as we prove both theorems.

Consider the $\mathbb{F}$-linear span of $f_{0}, \ldots, f_{n}$ as an $\mathbb{F}$-vector space, and partition

$$
\{0, \ldots, n\}=I_{0} \cup \cdots \cup I_{u-1} \quad \text { with } J_{j} \in I_{j} \text { for } j=0, \ldots, u-2
$$

as in Lemma 5.1. Let $J_{-1}=\emptyset$. Also, without loss of generality, assume $0 \in I_{0}$. Let $n_{j}$ be the cardinality of $I_{j}$ for $j=0, \ldots, u-1$. Note that $n_{0} \leq d+1$ by the minimality of $I_{0}$ and that $n_{j} \leq d$ for $j \geq 1$ by the minimality of $I_{j} \cup J_{j-1}$. Set

$$
\gamma^{0,0}=\gamma^{1,0}=\cdots=\gamma^{u-1,0}=\gamma^{0}=\gamma^{1}=\cdots=\gamma^{u-1}=(0, \ldots, 0) .
$$

The $f_{i}$ for $i$ in $I_{0} \backslash\{0\}$ are linearly independent over $\mathbb{F}$. Therefore by Lemma 5.2 and Theorem 3.5, there exist multi-indices $\gamma^{0,1}, \ldots, \gamma^{0, n_{0}-2}$ such that the generalized Wronskian $W_{0}$ formed by the $f_{i}$ with respect to these multi-indices for $i$ in $I_{0} \backslash\{0\}$ is not identically zero, and moreover,

$$
\left|\gamma^{0, i}\right| \leq\left|\gamma^{0, i-1}\right|+c \leq c i
$$

Note that if $n_{0}=2$, we simply let $W_{0}$ be the $f_{i}$ for the unique index $i$ in $I_{0}$ different from 0 . Similarly, for $j=1, \ldots, u-1$, there exist multi-indices $\gamma^{j, 1}, \ldots, \gamma^{j, n_{j}-1}$ such that the generalized Wronskian $W_{j}$ formed by the $f_{i}$ with respect to these multi-indices for $i$ in $I_{j}$ is not identically zero, and

$$
\left|\gamma^{j, i}\right| \leq\left|\gamma^{j, i-1}\right|+c \leq c i \text {. }
$$

The total number of multi-indices we get this way is

$$
n_{0}-2+n_{1}-1+\cdots+n_{u-1}-1=n-u .
$$

Write these multi-indices as $\gamma^{u}, \ldots, \gamma^{n-1}$ with

$$
\left|\gamma^{u}\right| \leq \cdots \leq\left|\gamma^{n-1}\right| \leq c(d-1),
$$


and note that

$$
\left|\gamma^{i}\right| \leq\left|\gamma^{i-1}\right|+c
$$

For each minimal index set $I_{0}, J_{0} \cup I_{1}, \ldots, J_{u-2} \cup I_{u-1}$, there is a linear dependence relation

$$
\sum_{i=0}^{n} c_{j, i} f_{i}=0
$$

with $c_{j, i}$ non-zero elements of $\mathbb{F}$ when $i$ is in $J_{j-1} \cup I_{j}$ and 0 otherwise. Of course, this also gives rise to the linear equations

$$
\sum_{i=0}^{n} c_{0, i} D^{\gamma^{0, q}} f_{i}=0, \quad q=0, \ldots, n_{0}-2,
$$

and

$$
\sum_{i=0}^{n} c_{j, i} D^{\gamma^{j, q}} f_{i}=0, \quad q=0, \ldots, n_{j}-1, j=1, \ldots, u-1 .
$$

Let $M$ be the $n \times(n+1)$-matrix whose entries are $c_{j, i} D^{\gamma^{j, q}} f_{i}$, where the columns are indexed by $i$ and the rows are indexed by $j$ and $q$. Note that the sum of each row of $M$ is zero. Let $\Delta_{i}$ denote the determinant of the matrix $M$ with the $i$ th column deleted. Because the $i$ th column is the negative of the sum of the other columns, $\Delta_{i}= \pm \Delta_{j}$. From the block nature of $M$,

$$
\Delta_{0}=C_{0} W_{0} \cdots W_{u-1},
$$

where $C_{0}$ is a constant obtained by multiplying the appropriate $c_{j, i}$ 's, and hence is non-zero. Thus, $\Delta_{i}$ is non-zero for all $i$, and up to a constant is the product of the generalized Wronskians $W_{j}$.

Define

$$
\begin{aligned}
a & =\left|\gamma^{n-1}\right| \leq c(d-1), \\
b & =\sum_{i=u}^{n-1}\left|\gamma^{i}\right| \geq \sum_{i=u}^{n-2} \max \left\{1,\left|\gamma^{n-1}\right|-c i\right\} \\
& \geq a\left\lceil\frac{a}{c}\right\rceil-\frac{\left\lceil\frac{a}{c}\right\rceil\left(\left\lceil\frac{a}{c}\right\rceil-1\right)}{2} c \geq a
\end{aligned}
$$

and

$$
\bar{a}=\sum_{i=1}^{\bar{k}-1}\left|\gamma^{n-i}\right| \leq c \sum_{i=1}^{\bar{k}-1}(d-i)
$$

where the first inequality in (17) follows from (14). Note also that

$$
b \geq \bar{a} \geq a \geq 1 \text {, }
$$


where $a \geq 1$ follows from the fact that not all the Wronskians $W_{j}$ can be $1 \times 1$, for if they were, we would have either all the $f_{i}$ constant or $\operatorname{gcd}\left(f_{0}, \ldots, f_{n}\right)$ $\neq 1$.

If $\mathbb{F}$ has characteristic $p>0$, let $\sigma$ be the largest integer such that

$$
p^{\sigma} \leq \max \left\{\gamma_{j}^{i}: u \leq i \leq n-1 \text { and } 1 \leq j \leq m\right\} \leq a,
$$

where $\gamma^{i}=\left(\gamma_{1}^{i}, \ldots, \gamma_{m}^{i}\right)$.

We claim that $F$ divides

$$
\Delta_{0} \prod_{i=0}^{n} G_{i} .
$$

Indeed, suppose that $P$ is an irreducible element of $\mathcal{E}_{m}$ which divides $f_{i}$ with exact multiplicity $e$. If $\mathbb{F}$ has characteristic zero, then $P^{\min \{e, a\}}$ divides $G_{i}$ by Proposition 2.5, and if $e>a$, then $P^{e-a}$ divides $W_{j}$, where $i \in I_{j}$, by Lemma 6.6 and (16). Now suppose char $\mathbb{F}=p$ and $p^{v}$ is the largest power of $p$ dividing $e$. If $p^{v} \leq p^{\sigma} \leq a$, then $P^{\min \{e, a\}}$ divides $G_{i}$ by Propositon 4.1 and in the case $e>a$, we find that $P^{e-a}$ divides $W_{j}$, where $i \in I_{j}$, again by Lemma 6.6 and (16). If $p^{v}>p^{\sigma}$, then $P^{e}$ divides $\Delta_{0}$ by Lemma 6.6 and (20). Thus in all cases, $P^{e}$ divides $W_{j} G_{i}$, which divides $\Delta_{0} G_{i}$ by (15). Therefore,

$$
\log |F|_{r} \leq \log \left|\Delta_{0}\right|_{r}+\sum_{i=0}^{n} \log \left|G_{i}\right|_{r}+O(1)
$$

by Corollary 2.2.

Let

$$
F_{0}=\prod_{i \in I_{0} \backslash\{0\}} f_{i}, \quad F_{j}=\prod_{i \in I_{j}} f_{i}, \quad j=1, \ldots, u-1 .
$$

For each $j=0, \ldots, u-1$, the quotient $W_{j} / F_{j}$ is the determinant of a matrix consisting of logarithmic derivatives, and so by Lemma 3.2,

$$
\begin{aligned}
\left|\frac{W_{0}}{F_{0}}\right|_{r} \leq-\left(\sum_{q=0}^{n_{0}-2}\left|\gamma^{0, q}\right|\right) \log r, \\
\left|\frac{W_{j}}{F_{j}}\right|_{r} \leq-\left(\sum_{q=0}^{n_{j}-1}\left|\gamma^{0, q}\right|\right) \log r \quad \text { for } j=1, \ldots, u-1 .
\end{aligned}
$$

Then

$$
\frac{f_{0} \Delta_{0}}{F}=C_{0} \cdot \frac{W_{0}}{F_{0}} \cdots \frac{W_{u-1}}{F_{u-1}}
$$

and hence

$$
\log \left|f_{0}\right|_{r}+\log \left|\Delta_{0}\right|_{r}-\log |F|_{r} \leq-b \log r+O(1)
$$

by Lemma 3.2 and (17). Similarly, if $i$ is any index in $I_{0}$, then we can write $W_{0}$ as a determinant involving $D^{\gamma^{0, q}} f_{l}$ for $l \neq i$, and so $f_{i} W_{0} / f_{0} F_{0}$ is also a 
sum of products of logarithmic derivatives, and hence

as well. Thus

$$
\log \left|\frac{f_{i} W_{0}}{f_{0} F_{0}}\right|_{r} \leq-\left(\sum_{q=0}^{n_{0}-2}\left|\gamma^{0, q}\right|\right) \log r
$$

$$
\log \left|f_{i}\right|_{r}+\log \left|\Delta_{0}\right|_{r}-\log |F|_{r} \leq-b \log r+O(1)
$$

for all $i$ in $I_{0}$. Now let $i$ be in $I_{1}$ and let $j$ be in $J_{0}$. Then, by a similar argument,

$$
\frac{f_{i} \Delta_{0}}{F}=C_{0} \cdot \frac{f_{j} W_{0}}{f_{0} F_{0}} \cdot \frac{f_{i} W_{1}}{f_{j} F_{1}} \cdot \frac{W_{2}}{F_{2}} \cdots \frac{W_{u-1}}{F_{u-1}},
$$

and $f_{j} W_{0} / f_{0} F_{0}$ and $f_{i} W_{1} / f_{j} F_{1}$ are both sums of products of logarithmic derivatives. Hence

$$
\log \left|f_{i}\right|_{r}+\log \left|\Delta_{0}\right|_{r}-\log |F|_{r} \leq-b \log r+O(1) .
$$

Continuing, we find that for all $i$,

$$
\log \left|f_{i}\right|_{r}+\log \left|\Delta_{0}\right|_{r}-\log |F|_{r} \leq-b \log r+O(1),
$$

whence

$$
\max _{0 \leq i \leq n} \log \left|f_{i}\right|_{r} \leq \log |F|_{r}-\log \left|\Delta_{0}\right|_{r}-b \log r+O(1) .
$$

Combining this with (21), we get

$$
\max _{0 \leq i \leq n} \log \left|f_{i}\right|_{r} \leq \sum_{i=0}^{n} \log \left|G_{i}\right|_{r}-b \log r+O(1) .
$$

If we use the Poisson-Jensen-Green type formula (2) and the definition of counting functions, this can also be written, for $r \geq 1$, as

$$
\max _{0 \leq i \leq n} \log \left|f_{i}\right|_{r} \leq \sum_{i=0}^{n} N_{G_{i}}(0, r)-b \log r+O(1),
$$

which is precisely (9).

We now show (12). Let $P$ be an irreducible element of $\mathcal{E}_{m}$ that divides $F$. By the hypotheses of the theorem, there is at least one $f_{i}$ such that $P$ does not divide $f_{i}$. Because, as above, we can write $\Delta_{0}$ as a product of Wronskians not involving $f_{i}$, we can use Lemma 6.7 to conclude by (18) that $P$ divides $\Delta_{0}$ with multiplicity at least $e-\bar{a}$. Thus, $F / \operatorname{gcd}\left(F, \Delta_{0}\right)$ divides $\operatorname{gcd}\left(F, S(F)^{\bar{a}}\right)$. Hence, by Proposition 2.2, the Poisson-Jensen-Green type formula (2), and the definition of truncated counting functions, for $r \geq 1$,

$$
\log |F|_{r}-\log \left|\Delta_{0}\right|_{r} \leq\left|\operatorname{gcd}\left(F, S(F)^{\bar{a}}\right)\right|_{r}+O(1)=N_{F}^{(\bar{a})}(0, r)+O(1) .
$$

Combining this with (22), we get for $r \geq 1$

which is (12).

$$
\max _{0 \leq i \leq n} \log \left|f_{i}\right|_{r} \leq N_{F}^{(\bar{a})}(0, r)-b \log r+O(1),
$$




\section{References}

[1] V. H. An, Height of p-adic holomorphic maps in several variables and applications, Acta Math. Vietnam. 27 (2002), 257-269.

[2] V. H. An and D. Q. Manh, p-Adic Nevanlinna-Cartan theorem in several variables for Fermat type hypersurfaces, East-West J. Math. 4 (2002), 87-99.

[3] - - - The "ABC" conjecture for p-adic entire functions of several variables, Southeast Asian Bull. Math. 27 (2004), 959-972.

[4] M. Bayat and H. Teimoori, A new bound for an extension of Mason's theorem for functions of several variables, Arch. Math. (Basel) 82 (2004), 230-239.

[5] M. de Bondt, Another generalization of Mason's ABC-theorem, arXiv: 0707.0434v1.

[6] S. Bosch, U. Güntzer and R. Remmert, Non-Archimedean Analysis, Grundlehren Math. Wiss. 261, Springer, Berlin, 1984.

[7] A. Boutabaa and A. Escassut, Nevanlinna theory in characteristic $p$ and applications, in: Analysis and Applications-ISAAC 2001 (Berlin), H. G. W. Begehr et al. (eds.), Int. Soc. Anal. Appl. Comput. 10, Kluwer, Dordrecht, 2003, 97-107.

[8] - , - Nevanlinna theory in positive characteristic and applications, Ital. J. Pure Appl. Math. 23 (2008), 45-66.

[9] J. Browkin and J. Brzeziński, Some remarks on the abc-conjecture, Math. Comp. 62 (1994), 931-939.

[10] W. D. Brownawell and D. Masser, Vanishing sums in function fields, Math. Proc. Cambridge Philos. Soc. 100 (1986), 427-434.

[11] W. Cherry, Existence of GCD's in rings of non-Archimedean entire functions, preprint.

[12] W. Cherry and Z. Ye, Non-Archimedean Nevanlinna theory in several variables and the non-Archimedean Nevanlinna inverse problem, Trans. Amer. Math. Soc. 349 (1997), 5043-5071.

[13] M. van Frankenhuysen, Hyperbolic spaces and the abc conjecture, Ph.D. thesis, Katholieke Universiteit Nijmegen, 1995.

[14] —, The ABC theorem for meromorphic functions, arXiv: 0805.1729v1.

[15] H. Fujimoto, Nonintegrated defect relation for meromorphic maps of complete Kähler manifolds into $P^{N_{1}}(C) \times \cdots \times P^{N_{k}}(C)$, Japan. J. Math. (N.S.) 11 (1985), 233-264.

[16] D. Goldfeld, Modular forms, elliptic curves and the ABC-conjecture, in: A Panorama of Number Theory or the View from Baker's Garden (Zürich, 1999), G. Wüstholz (ed.), Cambridge Univ. Press, Cambridge, 2002, 128-147.

[17] Hà Huy Khoái, La hauteur des fonctions holomorphes p-adiques de plusieurs variables, C. R. Acad. Sci. Paris Sér. I Math. 312 (1991), 751-754.

[18] L.-C. Hsia and J. T.-Y. Wang, The ABC theorem for higher-dimensional function fields, Trans. Amer. Math. Soc. 356 (2004), 2871-2887.

[19] P.-C. Hu and C.-C. Yang, The "abc" conjecture over function fields, Proc. Japan Acad. Ser. A Math. Sci. 76 (2000), 118-120.

[20] - - - A note on Browkin-Brzeziński conjecture, in: Ultrametric Functional Analysis, B. Diarra et al. (eds.), Contemp. Math. 384, Amer. Math. Soc., Providence, RI, 2005, 101-109.

[21] S. Lang, Old and new conjectured Diophantine inequalities, Bull. Amer. Math. Soc. (N.S.) 23 (1990), 37-75.

[22] R. C. Mason, Diophantine Equations over Function Fields, London Math. Soc. Lecture Note Ser. 96, Cambridge Univ. Press, Cambridge, 1984.

[23] —, Norm form equations. I, J. Number Theory 22 (1986), 190-207. 
[24] J. Noguchi and T. Ochiai, Geometric Function Theory in Several Complex Variables, Transl. Math. Monogr. 80, Amer. Math. Soc., Providence, RI, 1990.

[25] J. Oesterlé, Nouvelles approches du "théorème" de Fermat, Séminaire Bourbaki 1987/88. Astérisque 161-162 (1988), Exp. No. 694, 4, 165-186.

[26] N. T. Quang and P. D. Tuan, A note on Browkin-Brzeziński's conjecture, Int. J. Contemp. Math. Sci. 2 (2007), 1335-1340.

[27] K. F. Roth, Rational approximations to algebraic numbers, Mathematika 2 (1955), $1-20$.

[28] B. V. Shabat, Distribution of Values of Holomorphic Mappings, Transl. Math. Monogr. 61, Amer. Math. Soc., Providence, RI, 1985.

[29] H. N. Shapiro and G. H. Sparer, Extension of a theorem of Mason, Comm. Pure Appl. Math. 47 (1994), 711-718.

[30] C. L. Stewart and K. Yu, On the abc conjecture, II, Duke Math. J. 108 (2001), 169-181.

[31] W. W. Stothers, Polynomial identities and Hauptmoduln, Quart. J. Math. Oxford Ser. (2) 32 (1981), 349-370.

[32] L. N. Vaserstein, Quantum (abc)-theorems, J. Number Theory 81 (2000), 351-358.

[33] P. Vojta, Diophantine Approximations and Value Distribution Theory, Lecture Notes in Math. 1239, Springer, Berlin, 1987.

[34] J. F. Voloch, Diagonal equations over function fields, Bol. Soc. Brasil. Mat. 16 (1985), no. 2, 29-39.

[35] U. Zannier, Some remarks on the S-unit equation in function fields, Acta Arith. 64 (1993), 87-98.

Department of Mathematics

University of North Texas

1155 Union Circle \#311430

Denton, TX 76203-5017, U.S.A.

E-mail: wcherry@unt.edu
Department of Mathematics and Statistics

MSC03 2150

1 University of New Mexico Albuquerque, NM 87131-0001, U.S.A. E-mail: ctoropu@hotmail.com

Received on 23.7.2008

and in revised form on 29.8.2008 